\title{
Monotone multiscale finite volume method
}

\author{
Yixuan Wang ${ }^{1}$ Hadi Hajibeygi ${ }^{2}$ - Hamdi A. Tchelepi ${ }^{1}$
}

Received: 14 August 2014 / Accepted: 19 May 2015 / Published online: 16 August 2015

(C) The Author(s) 2015. This article is published with open access at Springerlink.com

\begin{abstract}
The MultiScale Finite Volume (MSFV) method is known to produce non-monotone solutions. The causes of the non-monotone solutions are identified and connected to the local flux across the boundaries of primal coarse cells induced by the basis functions. We propose a monotone MSFV (m-MSFV) method based on a local stencil-fix that guarantees monotonicity of the coarse-scale operator, and thus, the resulting approximate fine-scale solution. Detection of non-physical transmissibility coefficients that lead to non-monotone solutions is achieved using local information only and is performed algebraically. For these 'critical' primal coarse-grid interfaces, a monotone local flux approximation, specifically, a Two-Point Flux Approximation (TPFA), is employed. Alternatively, a local linear boundary condition can be used for the dual basis functions to reduce the degree of non-monotonicity. The local nature of the two strategies allows for ensuring monotonicity in local sub-regions, where the non-physical transmissibility occurs. For practical applications, an adaptive approach based on
\end{abstract}

Hadi Hajibeygi

H.Hajibeygi@tudelft.nl

Yixuan Wang

yixuanw@stanford.edu

Hamdi A. Tchelepi

tchelepi@stanford.edu

1 Department of Energy Resources Engineering, Stanford University, 367 Panama St., Rm. 065, Stanford, CA 94305-2220, USA

2 Department of Geoscience and Engineering, Faculty of Civil Engineering and Geosciences, TU Delft, P.O. Box 5048, 2600 GA Delft, The Netherlands normalized positive off-diagonal coarse-scale transmissibility coefficients is developed. Based on the histogram of these normalized coefficients, one can remove the large peaks by applying the proposed modifications only for a small fraction of the primal coarse grids. Though the m-MSFV approach can guarantee monotonicity of the solutions to any desired level, numerical results illustrate that employing the m-MSFV modifications only for a small fraction of the domain can significantly reduce the nonmonotonicity of the conservative MSFV solutions.

Keywords Multiscale finite volume method - Iterative multiscale methods - Algebraic multiscale solver - Scalable linear solvers - Monotone flux approximation schemes . Multipoint flux approximation · Porous media

\section{Introduction}

Increasing demand for efficient and accurate simulation of multiphase flow in large-scale heterogeneous porous media has motivated the development and extension of the MultiScale methods [1-9]. Among the proposed multiscale methods, the Mixed MultiScale Finite-Element (MMSFE) $[5,7,8]$ and the multiscale finite volume (MSFV) [6] methods provide locally mass-conservative solutions, which is a crucial property for solving coupled flow and transport problems. Compared to MMSFE, MSFV has the advantage of solving the flow problems with less unknowns and is quite applicable to reservoir simulation practices. Recent developments of the MSFV method allow for compositional effects and complex wells, making it a promising approach for the next-generation of reservoir flow simulators [10-20].

For a wide range of heterogeneous test cases, the MSFV results are shown to be in good agreement with reference 


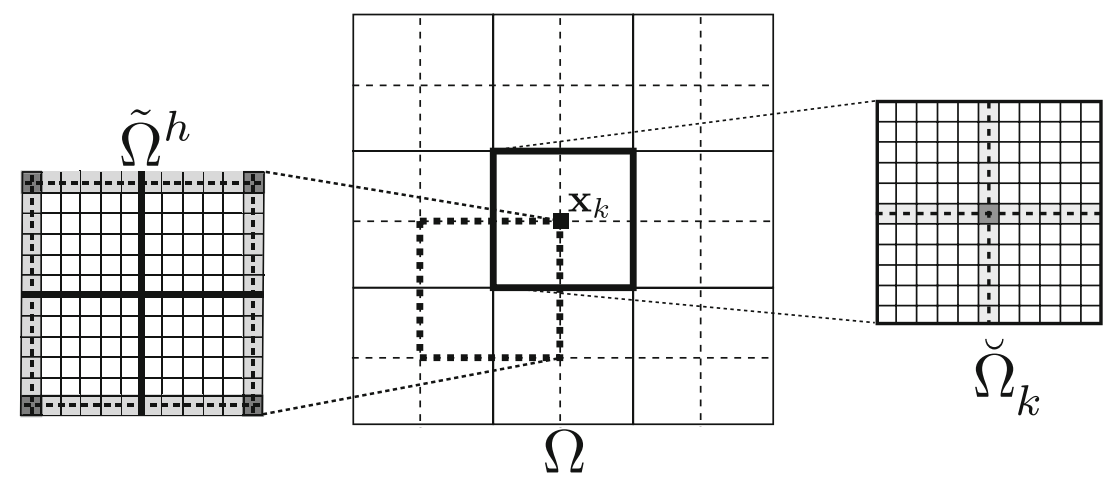

Fig. 1 Illustration of the MSFV primal- and dual-coarse grids imposed on the given $2 \mathrm{D}$ computational domain $\Omega$. The figure also shows the detail of a primal-coarse $\breve{\Omega}_{k}$ (coarse control volume) and a

fine-scale solutions, however, come with strong sensitivity to large contrasts in the local permeability and anisotropy in the transmissibility. To improve the quality of the reconstructed fine-scale solution, iterative MSFV (i-MSFV) strategies have been developed [21-23]. In the development of the Algebraic Multiscale Solver (AMS) [23], the coarse-scale symmetric-positive-definite system of MSFE is used to reduce the error norm to arbitrarily small values, while MSFV is employed only at the final stage to obtain a conservative velocity field. Having a conservative velocity field is a critical requirement for solving the nonlinear transport equations accurately and efficiently. Moreover, local mass conservation allows for adaptive computations and the use of relatively loose tolerances as a function of time [20, 21, 24]. Thus, in the context of a multiscale linear solver, the final step of using MSFV to ensure local conservation must be performed in a manner that minimizes the degree of nonmonotonicity in the reconstructed fine-scale pressure solution. To improve the quality of the MSFV solutions for slightly heterogeneous and grid-aligned anisotropic coefficients, a Compact-MSFV (C-MSFV) operator was proposed [25]. While the C-MSFV was effective for many grid-aligned anisotropic problems, it does not overcome the problem with nonmonotonicity for highly heterogeneous anisotropic fields. For heterogeneous problems, some improvements were observed by changing the boundary conditions (BC) for all local problems [26].

In this work, the cause of the non-physical peaks associated with the MSFV operator for highly heterogeneous problems is identified clearly and resolved. The peaks are associated with the discretization stencil of coarse nodes that are surrounded by low-permeability regions. It is shown that for these critical coarse nodes, integration of the flux induced by the dual basis functions can result in negative transmissibilities for the coarse-scale pressure system. A monotone MSFV (m-MSFV) method is devised on the basis of a local stencil-fix approach, which guarantees the dual-coarse $\tilde{\Omega}^{h}$ (local domain) cells, respectively. A coarse node, $\boldsymbol{x}_{k}$, here chosen the central cell inside $\breve{\Omega}_{k}$, is also shown. Boundary cells of local domains (dual-coarse cells) are also highlighted in gray

monotonicity of the MSFV solution. The critical interfaces with non-physical transmissibility values for the coarsescale system are detected algebraically. Then, a local TwoPoint-Flux-Approximation (TPFA) scheme is used to calculate the coarse-scale entries for the critical coarse faces only. In addition, the Linear Boundary Condition (LBC) can be employed for the basis function calculations of the critical regions. The LBC-based $\mathrm{m}-\mathrm{MSFV}$ reduces the norm of non-physical peaks (reducing nonmonotonicity). In contrast to the TPFA-based approach, however, the LBC-based m-MSFV cannot remove all the negative (non-physical) transmissibilies from the coarse-scale system.

The local nature of m-MSFV allows it to be employed adaptively in space and time. In this paper, a histogram of the critical interfaces is calculated based on a normalized value of the non-physical transmissibility coefficients. Then, based on a threshold value, only critical interfaces with large values are detected and fixed. This thresholdbased approach allows for a reasonable trade-off between the accuracy and monotonicity of the solutions.

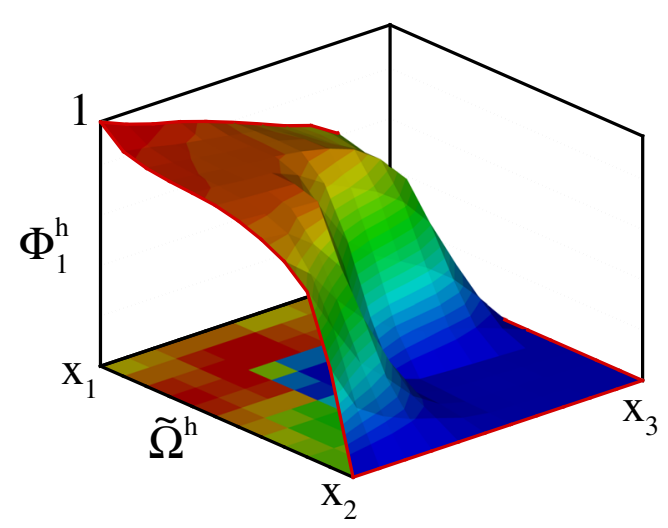

Fig. 2 Illustration of the basis function $\Phi_{1}^{h}$ solved on dual-coarse cell $\tilde{\Omega}^{h}$ subject to the reduced boundary condition. Note that the basis functions are always monotone and satisfy $0 \leq \Phi_{k}^{h} \leq 1$, provided that the mobility tensor $\lambda$ is positive definite 


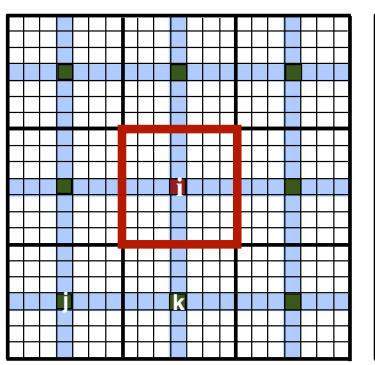

$\square$ Fine cells $\square$ Dual-coarse edge cells
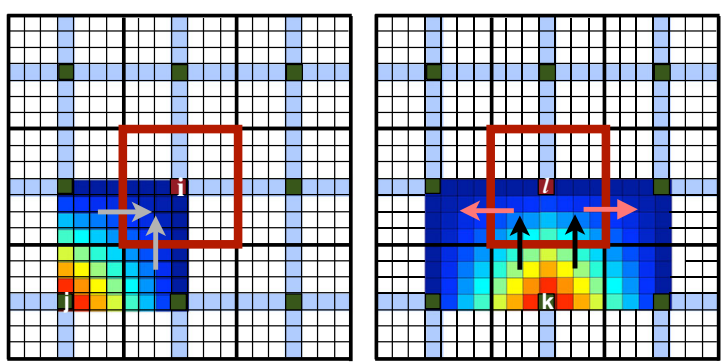

$\square$ Coarse-node cells
Fig. 3 (left): Illustration of a $3 \times 3$ coarse- and $21 \times 21$ fine- grid domain. The coarse cell $i$ is highlighted in red, neighboring $k$ and $j$ on its South and South-West sides. Also shown are the induced fluxes by

The paper proceeds as follows. After a short review of the MSFV method, in Section 2, the MSFV coarse-scale operator (system) is described in detail. In Section 3, the $\mathrm{m}-\mathrm{MSFV}$ method is presented. Numerical results are shown in Section 4, followed by Section 5 where the paper is concluded.

\section{Multiscale finite volume method}

The elliptic equation for pressure, $p$, can be written as

$-\nabla \cdot(\lambda \cdot \nabla p)=q$,

where the highly heterogeneous mobility (assumed diagonal) tensor and the source terms are denoted with $\lambda$ and $q$, respectively. The problem (1) is well posed for a $d$ dimensional computational domain $\Omega \subset \Re^{d}$, subject to proper boundary condition at $\partial \Omega \subset \mathfrak{R}^{d-1}$. The discrete form of Eq. 1 at the given fine-scale (denoted here on by superscript $f$ ), where the coefficients $\lambda$ are computed using a finite-volume TPFA scheme [27], can be written as

$A^{f} p^{f}=q^{f}$,

where entries of the transmissibility matrix $A^{f}$ are $\left.a_{i j}^{f}\right|_{i \neq j}=-\frac{\bar{\lambda}_{i j} \cdot \mathbf{n}_{i j}}{\delta x_{i j}} \cdot \mathbf{n}_{i j} \delta A_{i j}$. Here, $\bar{\lambda}_{i j}, \delta A_{i j}$, and $\delta x_{i j}$ are the harmonically averaged permeability, differential element cross section area and the distance between the computational nodes $i$ and $j$, respectively. Also, the normal unit vector $\mathbf{n}_{i j}$ points out of volume $i$ at its cross section with cell $j$. Note that $a_{i j}^{f}=a_{j i}^{f}$ and $a_{i i}^{f}=-\sum_{j=1, j \neq i}^{N_{f}} a_{i j}^{f}$, the $\Phi_{j}$ (middle) and $\Phi_{k}$ (right). Note that only the overlapping part of the basis functions are plotted, and that for simplicity of the illustration a homogeneous problem is used

where $N_{f}$ is the number of fine-scale finite volumes. In our implementation, the positive definite mobility tensor leads to non-positive off-diagonal $\left(\left.a_{i j}^{f}\right|_{i \neq j} \leq 0\right)$ and non-negative diagonal $\left(a_{i i}^{f} \geq 0\right)$ entries for the transmissibility matrix.

The MSFV method employs primal- $\left(\breve{\Omega}^{c}\right)$ and dual- $\left(\tilde{\Omega}^{h}\right)$ coarse grids superimposed on the given fine grid (Fig. 1). The fine-scale pressure field is constructed as follows:

$p^{f} \approx p^{M S}=\sum_{k=1}^{N_{c}} \Phi_{k} \breve{p}_{k}$,

where $N_{c}$ represents the number of coarse-scale control volumes. The locally computed basis functions $\Phi_{k}$ are used to prolong the coarse-scale solution $\breve{p}_{k}$ onto the fine-scale resolution. Basis functions are first computed on dual-coarse cells, $\tilde{\Omega}^{h}$, as illustrated in Fig. 2 , and then assembled for all dual cells, $N_{d}$, i.e., $\Phi_{k}=\sum_{h=1}^{N_{d}} \Phi_{k}^{h}$. Note that the precomputed correction term at the fine-scale, $\Psi$, can be used to improve this approximation, leading to $p^{f} \approx p^{\prime}=p^{M S}+$ $\Psi$. The correction term is an independent stage to improve the multiscale solution, $p^{M S}$. Note that the correction term does not modify the coarse-scale system matrix; hence, we do not consider it in our analysis. For more detailed analysis of the correction term, we refer to [23].

The basis functions are local solutions of the governing Eq. 1, i.e.,

$$
\begin{array}{rlr}
-\nabla \cdot\left(\lambda \cdot \nabla \Phi_{k}^{h}\right) & =0 \quad \text { on } \quad \begin{array}{c}
\tilde{\Omega}^{h} \\
\Phi_{k}^{h}\left(x_{i}\right)=\delta_{k i}
\end{array} \quad \forall x_{i},
\end{array}
$$

Fig. 4 Logarithm of permeability field for SPE 10 bottom layer [28]. The domain consists of $220 \times 60$ fine- (not shown) and $20 \times 12$ coarse(shown) grid cells. Two subdomains of the size $3 \times 3$ coarse cells are highlighted

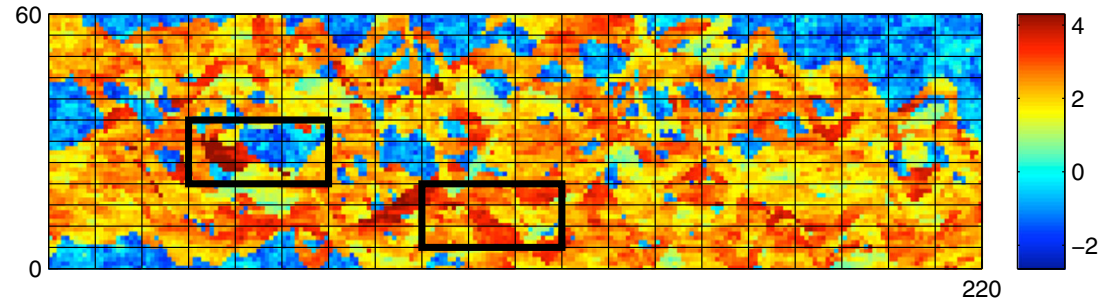


Fig. 5 (top-left): Logarithm of permeability field with coarse grid and coarse nodes, extracted from Fig 4. (top-right): part of the basis function $\Phi_{k}$ overlapping with coarse cell $i$ (coarse cell (10,3) in Fig. 4). (bottom-left): basis function $\Phi_{i}$; (bottom-right): superimposed MSFV pressure field, $p^{M S}=\sum \Phi_{k} \breve{p}_{k}$, obtained for $\Omega^{h 1}$
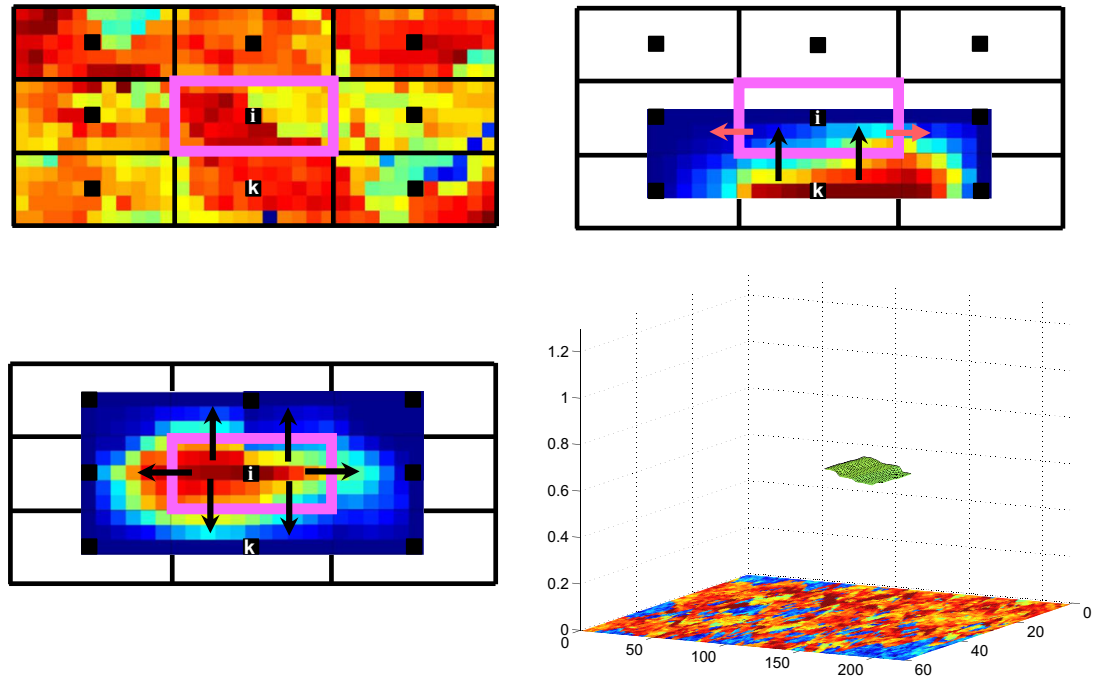

where $\delta_{k i}$ is the Dirac delta function, i.e., $\left.\delta_{k i}\right|_{k=i}=1$ and $\left.\delta_{k i}\right|_{k \neq i}=0$. Equation 4 subject to Eq. 5 at the corner vertices is solvable if a proper BC is imposed on $\partial \tilde{\Omega}^{h}$. The reduced-dimensional problem condition can be stated as

$-\nabla_{\perp} \cdot\left(\lambda \cdot \nabla \Phi_{k}^{h}\right)_{\perp}=0 \quad$ at $\quad \partial \tilde{\Omega}^{h}$,

which has been widely used in the multiscale literature. The subscript $\perp$ denotes the normal projection (operator or vector) with respect to the boundary. Alternatively, if one ignores the mobility variation along the boundary, i.e., $\boldsymbol{\lambda}=\boldsymbol{I}$ at $\partial \tilde{\Omega}^{h}$, the formulation reduces to the LBC. Note that the basis functions computed with either of the two local $\mathrm{BC}$ are monotone with numerical values between 0 and 1 , i.e., $0 \leq \Phi_{k}(\mathbf{x}) \leq 1 \quad \forall \mathbf{x} \in \Omega, k=\left\{1,2, \ldots, N_{c}\right\}$, provided that the fine-scale mobility coefficients $\lambda$ are positive definite. Therefore, in the superposition $p^{M S}=\sum \Phi_{k} \breve{p}_{k}$, $p^{M S}$ would violate the monotonicity property if and only if the $\breve{p}_{k}$ violates this property. Hence, all the non-physical peaks are associated with non-physical $\breve{p}_{k}$ values. This important fact guides us to the cause of the non-physical peaks in the MSFV solution, $p^{M S}$. That is, the properties of the coarse-scale system control the monotonicity behavior.

The superposition expression is substituted into Eq. 1 and integrated over coarse-control volume boundaries. After applying the Gauss integral rule, one obtains the coarsescale system as

$A^{c} \breve{p}=\int_{\breve{\Omega}} q d \Omega$,

where the coarse-scale transmissibility matrix entries $a_{i j}^{c}$ are

$a_{i j}^{c}=-\int_{\partial \breve{\Omega}_{i}}\left(\lambda \cdot \nabla \Phi_{j}\right) \cdot \mathbf{n}_{i} d \Gamma$.
Fig. 6 (top-left): Logarithm of permeability field with coarse grid and coarse nodes, extracted from Fig $4, \Omega^{h 2}$. (top-right): part of the basis function $\Phi_{k}$ overlapping with coarse cell $i$ (coarse cell $(5,6)$ in Fig. 4). (bottom-left): basis function $\Phi_{i}$; (bottom-right): superimposed MSFV pressure field, $p^{M S}=\sum \Phi_{k} \breve{p}_{k}$, obtained for $\Omega^{h 2}$. Note that a non-physical positive off-diagonal value of $a_{i k}^{c}=222.5$ and small positive value of $a_{i i}^{c}=0.65$ are calculated for coarse-system entries, which also clearly shows the $i$-th coarse-system row is not diagonally dominant
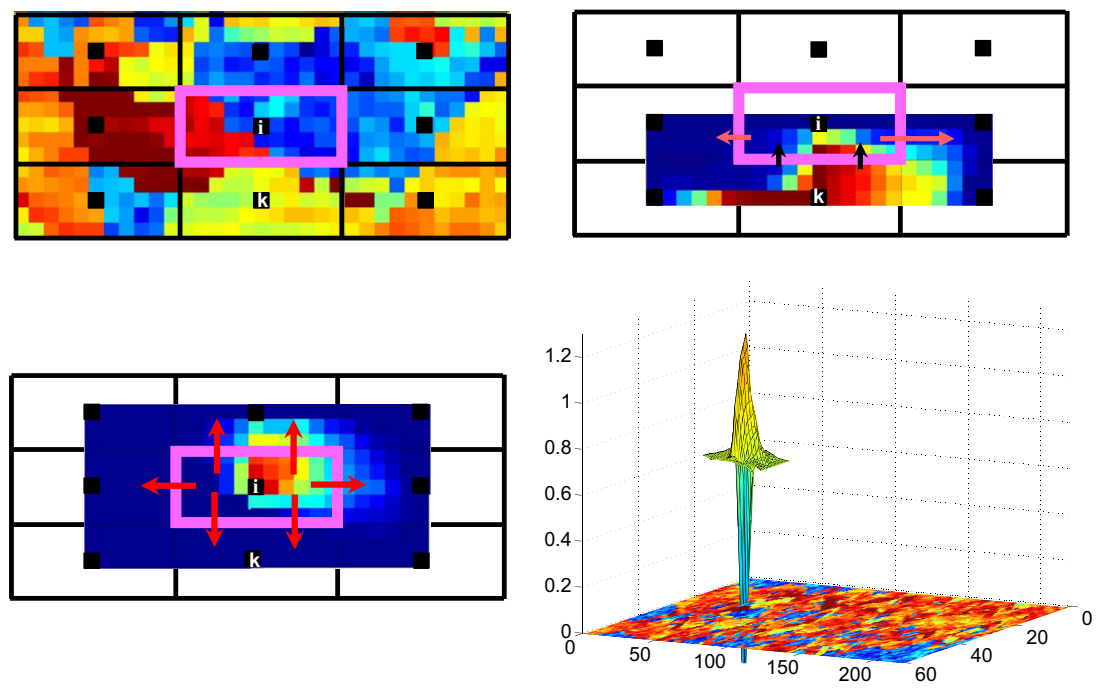
Fig. 7 Fine-scale reference (left) and MSFV (right) solutions for the SPE 10 bottom layer heterogeneous test case. There exist $220 \times 60$ fine- and $20 \times 12$ coarse- grid cells. Note that the MSFV superimposed solution (right) entails several non-physical peaks. The permeability field is also partly shown in the plots under the pressure solution
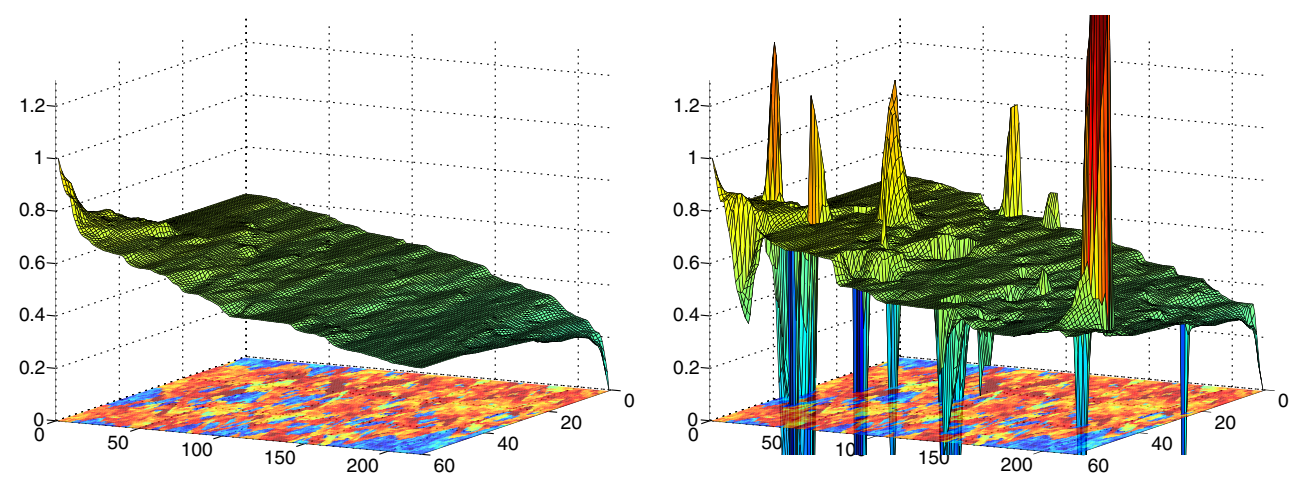

Here, $\mathbf{n}_{i}$ is the unit normal vector pointing out of the control volume (coarse-cell) $i$. Note that $\Phi_{j}=\sum_{h=1}^{N_{d}} \Phi_{j}^{h}$. Mass conservation leads to

$a_{i i}^{c}=-\sum_{j=1, i \neq j}^{N_{c}} a_{i j}^{c}=-\int_{\partial \check{\Omega}_{i}}\left(\lambda \cdot \nabla \Phi_{i}\right) \cdot \mathbf{n}_{i} d \Gamma$,

since $\sum_{j=1}^{N_{c}} \Phi_{j}^{h}=1$. Note that the coarse-scale system in MSFV is not guaranteed to be symmetric, i.e.,

$a_{i j}^{c}=-\int_{\partial \breve{\Omega}_{i}}\left(\lambda \cdot \nabla \Phi_{j}\right) \cdot \mathbf{n}_{i} d \Gamma \neq a_{j i}^{c}=-\int_{\partial \check{\Omega}_{j}}\left(\lambda \cdot \nabla \Phi_{i}\right) \cdot \mathbf{n}_{j} d \Gamma$,

since the coefficients are integrals of different functions over different control volume boundaries. This is in contrast to the symmetric-positive-definite MSFE coarse-scale operator. A coarse-scale system that has positive-definite mobility tensors at the fine scale is expected to yield negative off-diagonal, $a_{i j}^{c} \leq 0$, and positive diagonal, $a_{i i}^{c} \geq$ 0 values. Next, we study the integrals (8) and (9) and investigate the situations that may violate these conditions.

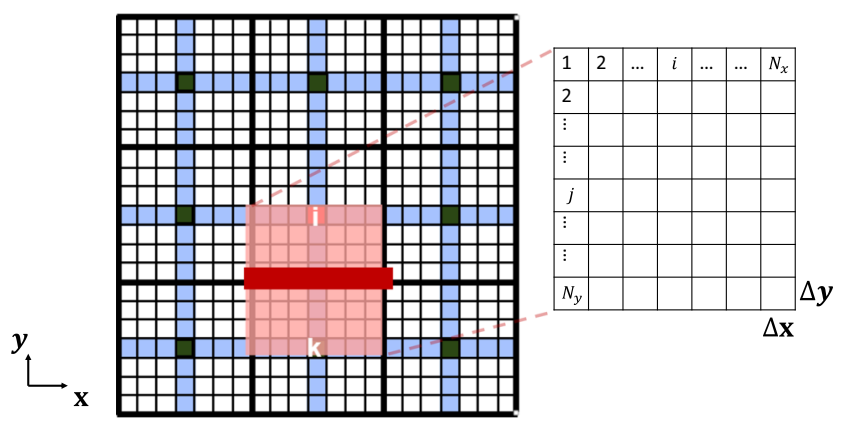

Fig. 8 Automatically detected critical interface (shown in bold red) where $a_{i k}^{c} \not \leq 0$. The highlighted region with a pink rectangle shows the local domain, where the transmissibility is calculated using summation of harmonically averaged values to replace with $a_{i k}^{c}$ and $a_{k i}^{c}$

\subsection{Coarse-scale Transmissibility Coefficients}

In order to study the coarse-scale transmissibility coefficients, a $3 \times 3$ coarse-grid problem in $2 \mathrm{D}$ is considered and shown in Fig. 3. We study the transmissibility coefficients between cell $i$ and two of its neighboring cells $j$ and $k$.

For the South-West neighboring cell, $j$, the flux induced by the basis function $\Phi_{j}, a_{i j}^{c}$, satisfies the physical property of $a_{i j}^{c} \leq 0$ because both boundary segments of control volume $i$ experience incoming fluxes. Note that the net induced flux (for any heterogeneous field) from $j$ to $i$ is always nonnegative. On the other hand, the fluxes induced by the basis function $\Phi_{k}$ must be computed along many (four in 2D) overlapping segments. For cell $i$, some of these fluxes are incoming and some others are outgoing. For many heterogeneous cases, the net incoming flux to the control volume $i$ is positive, leading to a negative off-diagonal entry, which is desirable.

Figure 4 shows the SPE 10 bottom layer permeability field which consists of $220 \times 60$ fine cells. The MSFV coarse

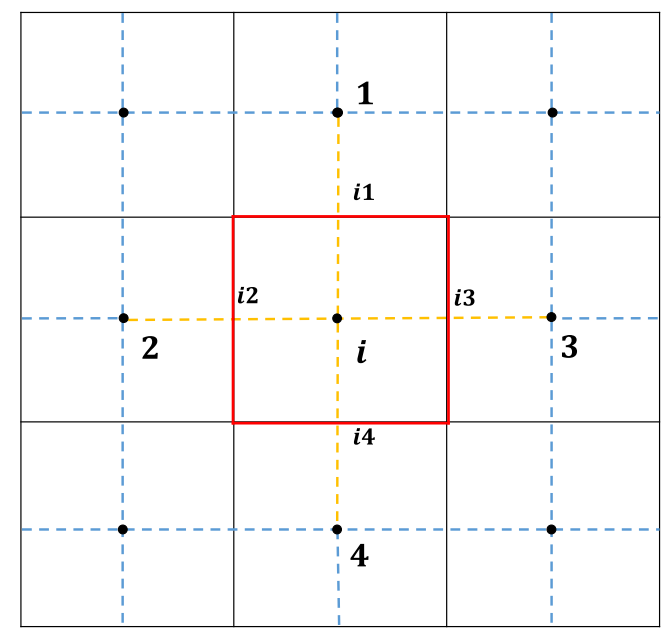

Fig. 9 Critical coarse node $i$ and its neighboring faces $F_{i j}$ (indicated by red solid lines) and edges $E_{i j}$ (indicated by yellow dash lines), $j=$ $1,2,3,4$ for 2D domain. The black lines indicate the coarse volumes 


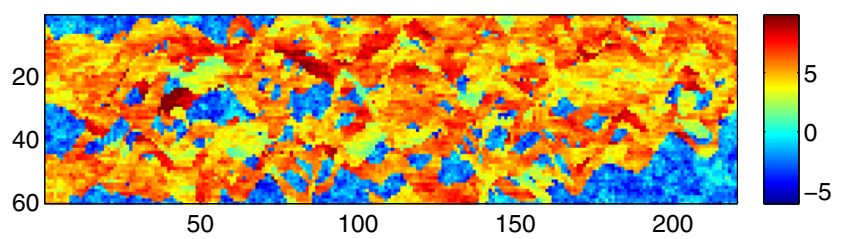

(a)

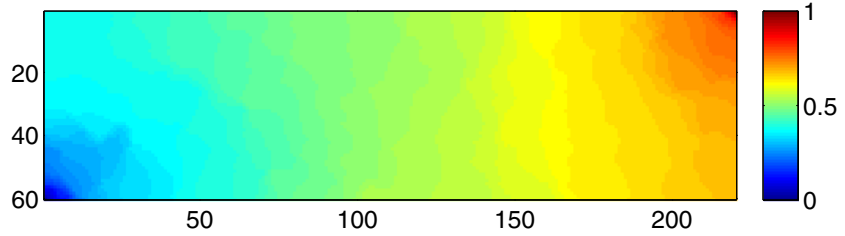

(b)

Fig. 10 Natural logarithm of the permeability (a) and fine-scale reference pressure (b) for the SPE 10 bottom layer

grid is also shown in the figure for a coarsening ratio of $11 \times 5$. Figure 5 shows an extracted rectangular subdomain from Fig. $4, \tilde{\Omega}^{h 1}$, between $(88,5) \leq(x, y) \leq(121,20)$. Figure 5 also shows that the central coarse cell $(10,3)$ of this subdomain has a net incoming flux induced by the basis function of its southern neighboring cell $(10,2)$, together with the interpolated pressure field only for the associated local domain, i.e., $p^{M S}$ in $\tilde{\Omega}^{h 1}$. To obtain this interpolated solution, a test case is solved subject to no-flow Neumann condition on all boundaries and Dirichlet condition of $p=1$ and $p=0$ at fine cells $(1,60)$ and $(220,1)$, respectively. Note that due to the positive diagonal and negative offdiagonal coarse-system entries corresponding to this local subregion, the interpolated solution is physical.

If for a heterogeneous field, the net incoming flux to the cell $i$ is negative, then off-diagonal entries $a_{i k}^{c}$ become positive. This situation happens when the coarse node $\boldsymbol{x}_{i}$ lies in a low-permeability region, compared with the other boundary cells between $i$ and $k$. There are other scenarios that would cause the same situation, e.g., if a shale layer (with very low permeability) crosses the boundary cells between $i$ and $k$. Note that in such cases, the reduced-problem local BC, between the cells $i$ and $k$, would lead to a solution with a constant value of one. This constant unity solution, which is then used as a Dirichlet condition for the internal cells, leads to a non-physical outgoing induced flux from the control volume. An example of such a case is illustrated in Fig. 6, where the domain $\Omega^{h 2}$ is extracted again from (and highlighted in) Fig. 4 for cells belonging to $(33,20) \leq(x, y) \leq$ $(66,35)$ interval. The integral incoming flux induced by $\Phi_{k}$ over the faces of the control volume $i$ is negative, which leads to a positive off-diagonal value of $a_{i k}^{c}=222.5$ for the coarse-scale system. The total outgoing fluxes induced by the basis function $\Phi_{i}$, over its own control volume is too small $\left(a_{i i}^{c}=0.65\right)$, which indicates that the corresponding row in the coarse-scale system is not diagonally dominant. This is closely related to the fact that the coarse node lies in a region with very low permeabilities. Note that the other cells (especially the boundary cells) have higher permeability values. As a result, the superimposed MSFV solution entails non-physical peaks (as shown in Fig. 6).

Figure 7, which is for the SPE 10 bottom layer, indicates that the original MSFV strategy leads to non-physical solutions at several locations. From this figure, it is clear that the

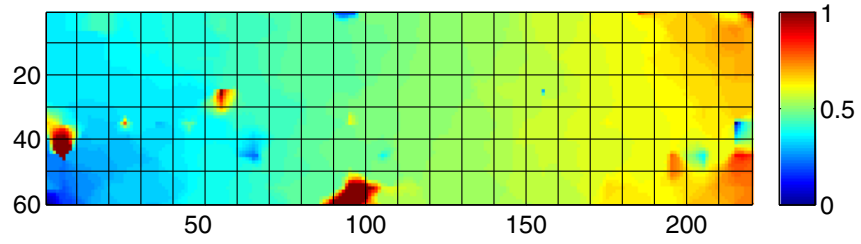

(a) Original $\operatorname{MSFV}\left(\left\|e_{p}\right\|_{2}=0.197 ;\left\|e_{p}\right\|_{\infty}=3.815\right)$

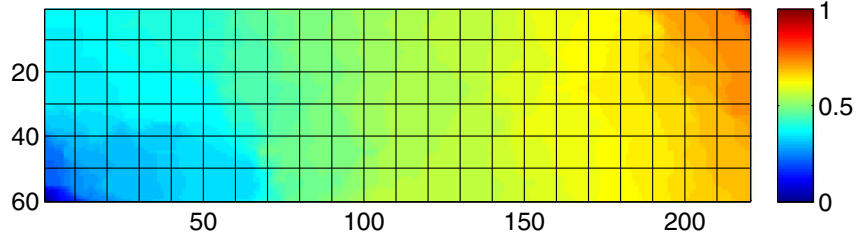

(b) $\mathrm{m}-\operatorname{MSFV}(\mathrm{TPFA})\left(\left\|e_{p}\right\|_{2}=0.035 ;\left\|e_{p}\right\|_{\infty}=0.071\right)$

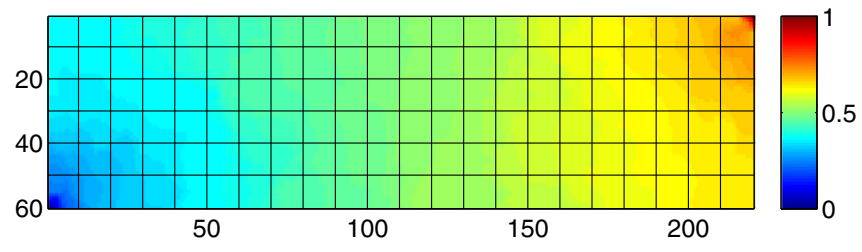

(c) $\operatorname{m}-\operatorname{MSFV}(\mathrm{LBC})\left(\left\|e_{p}\right\|_{2}=0.052 ;\left\|e_{p}\right\|_{\infty}=0.122\right)$

Fig. 11 Original MSFV and m-MSFV pressure solutions for the SPE 10 bottom layer, and the relative errors $e_{p}$. The coarse-scale grids are indicated by black lines 


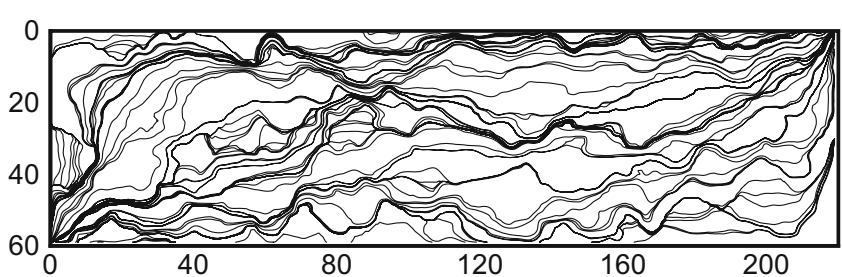

(a) Fine-scale reference

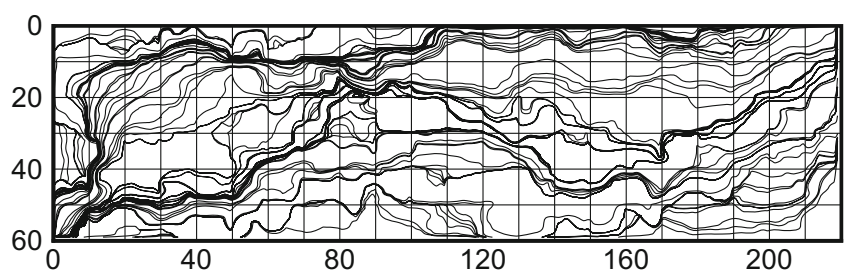

(c) m-MSFV (TPFA)

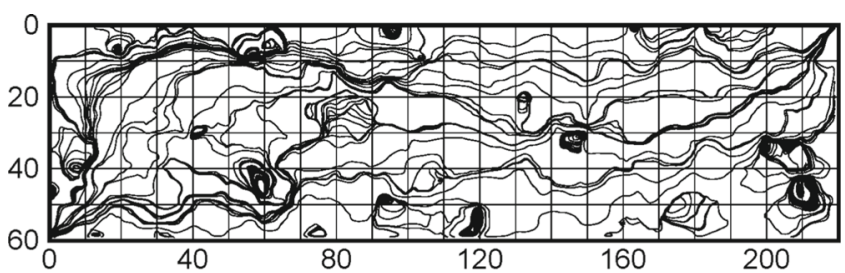

(b) Original MSFV

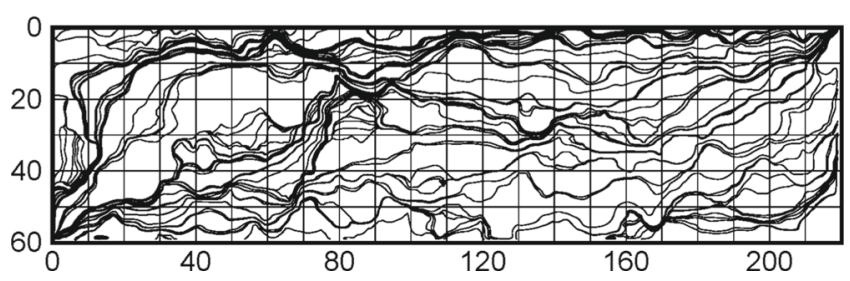

(d) m-MSFV (LBC)

Fig. 12 Streamline plots based on velocity fields reconstructed by fine-scale reference, original and m-MSFV pressure solutions for the SPE 10 bottom layer. The coarse-scale grids are indicated by black lines

peaks are located in regions with high contrasts in the permeability between the neighboring cells. In the next section, we describe a monotone MSFV method.

\section{Monotone MSFV (m-MSFV) method}

In this section, to ensure the monotonicity of the MSFV solution, two approaches are proposed.

Fig. 13 Histogram of $\eta_{i j}$ of the coarse-scale system $A_{c}$ for original MSFV (a), the reconstructed coarse-scale system for m-MSFV (TPFA) (b), and m-MSFV (LBC) (c), respectively

\subsection{Local TPFA approach}

This approach is based on local utilization of a physical flux calculation only for critical faces to ensure monotonicity of the MSFV solution. First, the coarse cell interfaces with negative transmissibility values, i.e., $a_{i k}^{c} \not \leq 0$, are detected. Then, instead of using the basis functions to provide the $a_{i k}^{c}$ values from Eq. 8, the transmissibility field between the cells $i$ and $k$ is calculated with TPFA which guarantees

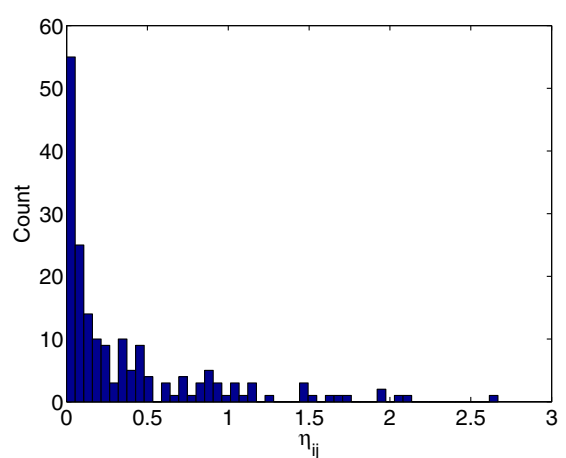

(a)

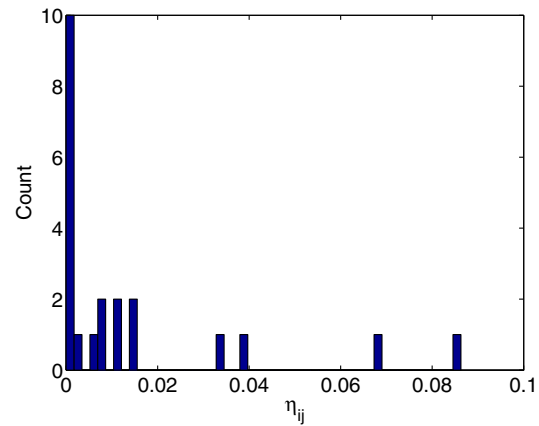

(b)

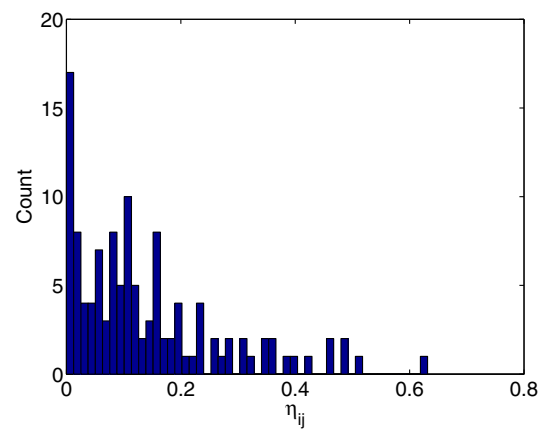

(c) 


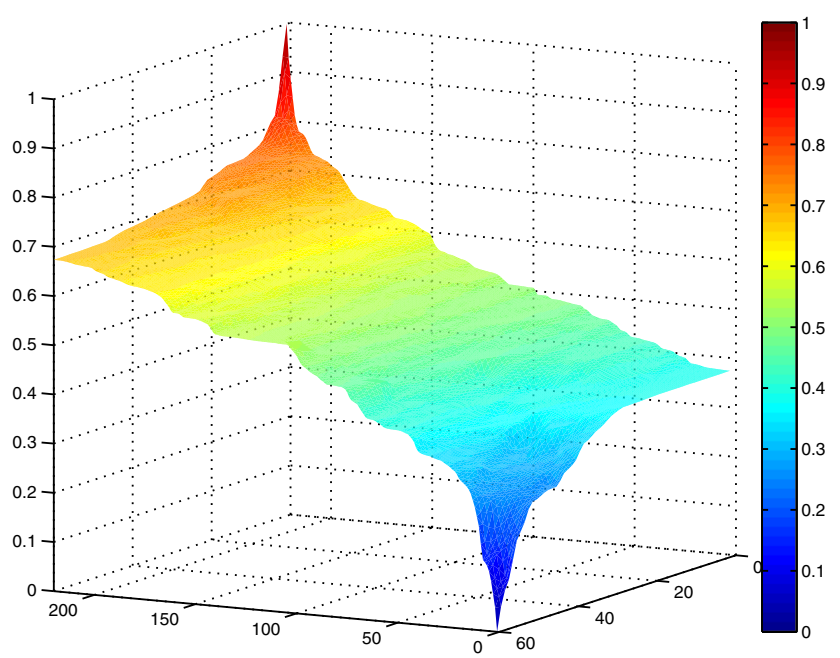

(a) Fine-scale reference

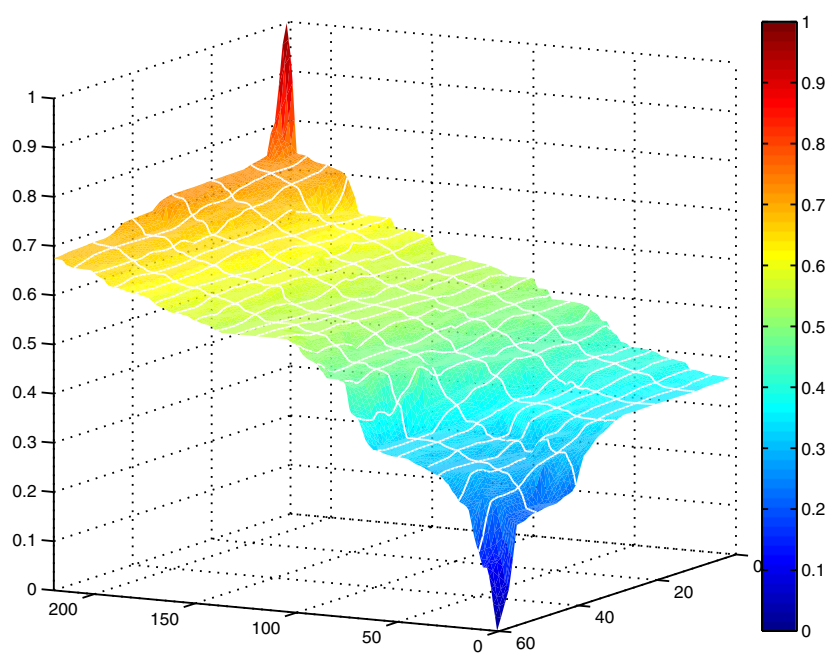

(c) m-MSFV (TPFA) with $\varepsilon=0$

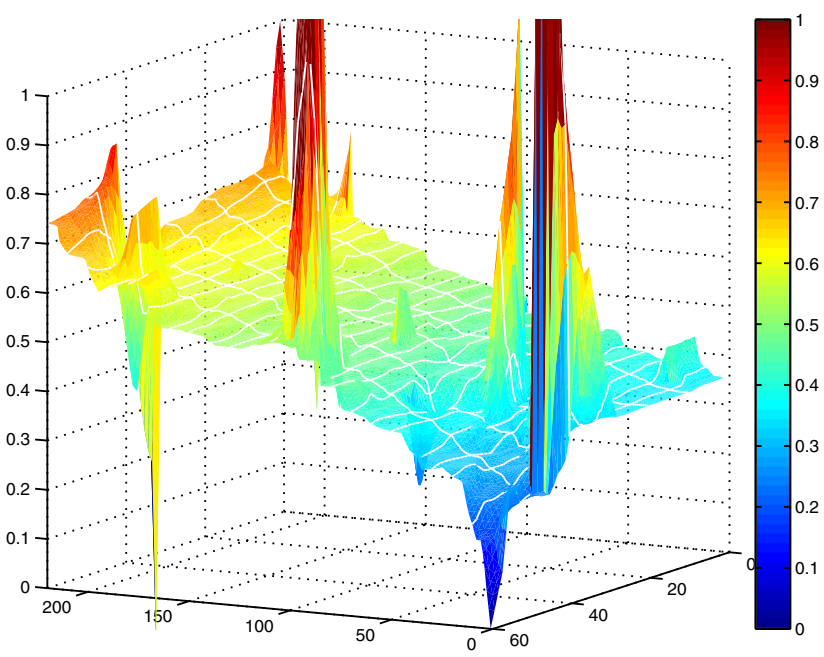

(b) Original MSFV

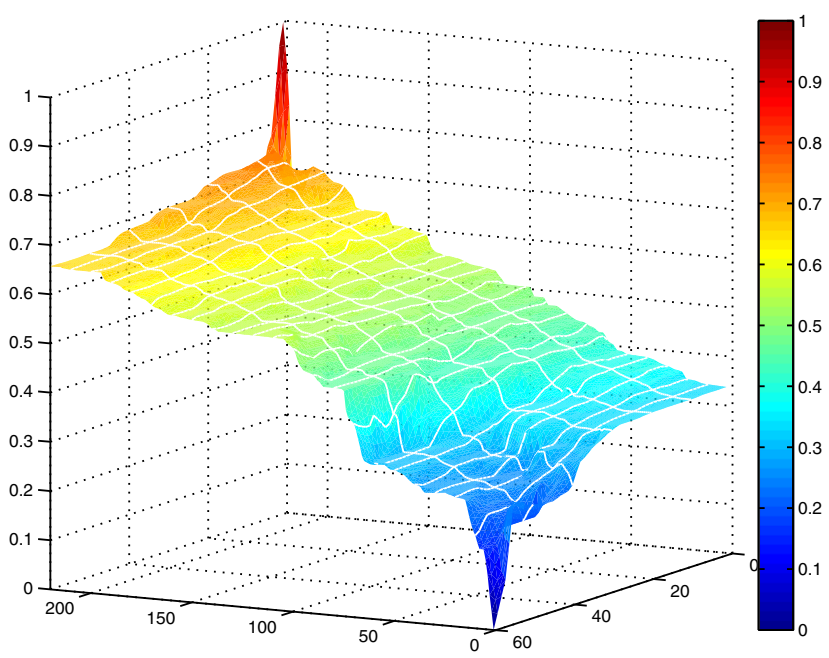

(d) m-MSFV (TPFA) with $\varepsilon=0.7$

Fig. 14 Pressure surface plots for fine-scale reference (a), original MSFV (b), m-MSFV (TPFA) with $\varepsilon=0(\mathbf{c})$ and $\varepsilon=0.7$ (d), respectively

that $a_{i k}^{c} \leq 0$. Figure 8 shows the highlighted pink region used to obtain an effective transmissibility coefficient at the interface between $i$ and $k$. The procedure to calculate TPFA-based coarse-scale transmissibility $T_{i k}^{c}$ is as follows. First, harmonically averaged transmissibility factors among columns of the highlighted pink cells are calculated. Then, the values are summed to compute $T_{i k}^{c}$. Therefore,

$T_{i k}^{c}=\sum_{i=1}^{N_{x}} \frac{1}{\sum_{j=1}^{N_{y}} \frac{1}{k_{i j}}} \frac{\Delta x}{\Delta y}$,

where $k_{i j}, \Delta x$, and $\Delta y$ represent fine-scale permeability, gridblock size in $x$ and $y$ directions, respectively.

To ensure conservation, the symmetric entry $a_{k i}^{c}$ is also updated with the same value as for the $a_{i k}^{c}$. Here, the new coarse-scale transmissibilities for the critical faces are computed based on averaging the fine-scale permeability field. Other options such as flow-based upscaling are also possible and can be incorporated into our monotone strategy, provided that they guarantee $a_{i k}^{c}<=0$. In this paper, we focus on our permeability-based strategy.

In fact, a slightly positive value $a_{i j}^{c}$ does not necessarily lead to non-monotone solutions, and only the $a_{i j}^{c}$ with relatively large positive values matter and have to be modified. In order to quantify the critical $a_{i j}^{c}$, an indicator $\eta_{i j}$ for each positive off-diagonal entry $a_{i j}^{c}$ of the coarse-scale coefficients matrix $A_{c}$ is used. We defined $\eta_{i j}=a_{i j}^{c} / \omega_{i}$, where $\omega_{i}$ represents the maximum absolute value of all the negative off-diagonal $a_{i j}^{c}$ in row $i$. The coarse node with an interface with $\eta_{i j}>\varepsilon$ is considered critical, where $\varepsilon$ is a user-specified threshold value. Then, all the neighboring 
interfaces associated with the critical coarse node are replaced by TPFA stencils. Algorithm 1 summarizes how the local TPFA approach is integrated in the MSFV procedure.

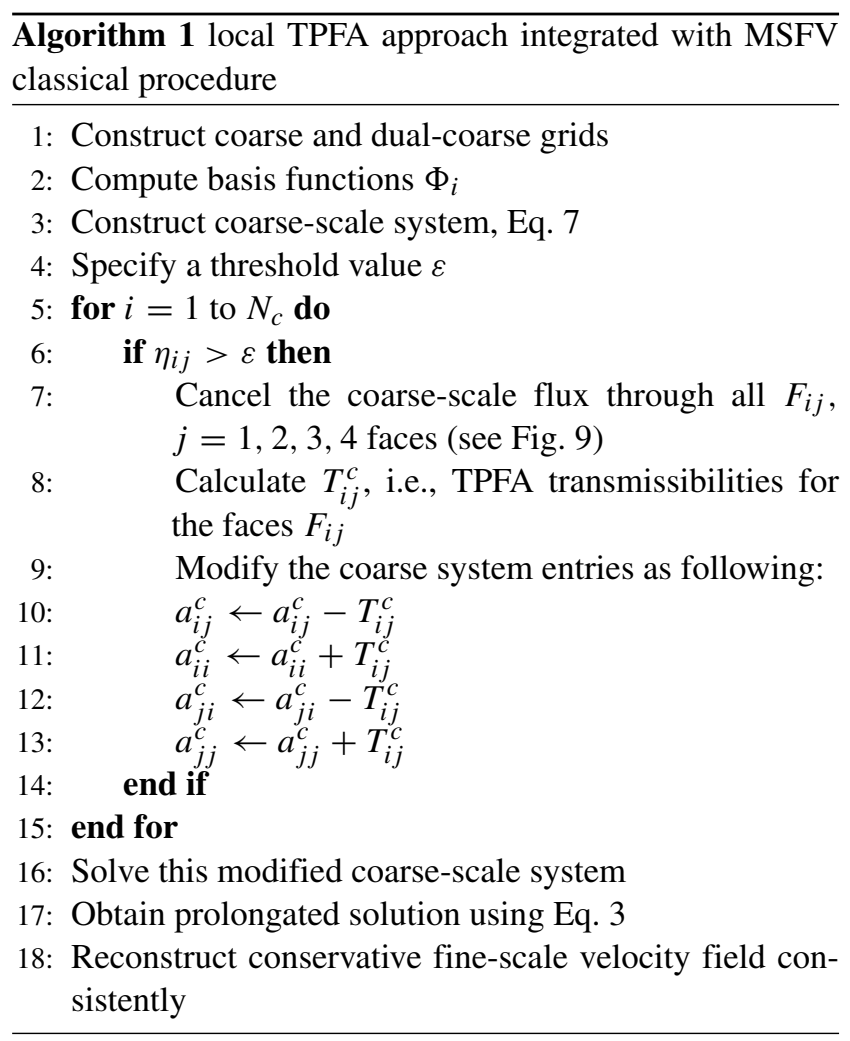

\subsection{Local linear BC approach}

In addition to the local TPFA approach, the nonmonotonicity of the MSFV pressure solution can be mitigated by locally using a Linear Boundary Condition (LBC) instead of the reduced BC. For the LBC approach, once the critical interface (i.e., the one with $\eta_{i j}>\varepsilon$ ) is detected, a linear $\mathrm{BC}$ is used for the corresponding dual coarse grid boundary crossing the detected interface. For the remaining boundaries, the reduced BC is used. Then, the basis functions affected by the linear $\mathrm{BC}$ are recomputed, and the coarse-scale system is reconstructed. Afterwards, the finescale solution is obtained by interpolating the coarse-scale solution with the modified basis functions. Finally, the conservative fine-scale velocity field can be constructed similar as in the classical MSFV method.

The local TPFA approach guarantees monotonicity of the solution, since the TPFA flux is used over the coarse interfaces. The local LBC approach reduces the degree of non-monotonicity; however, it cannot guarantee a monotone solution. In addition, the choice of the threshold value, $\varepsilon$, is a trade-off between the computational effort, quality of the solution, and the degree of monotonicity in the pressure field.

\section{Numerical results}

In this section, several test cases are solved to illustrate the proposed m-MSFV method. To quantify the accuracy of $\mathrm{m}$ MSFV, relative errors of pressure, velocity, and residuals, in terms of $L^{2}$ and $L^{\infty}$ norms, are used. These norms are defined as

$\left\|e_{p}\right\|=\left\|p^{m}-p^{f}\right\| /\left\|p^{o}-p^{f}\right\|$,

$\left\|e_{v}\right\|=\left\|v^{m}-v^{f}\right\| /\left\|v^{o}-v^{f}\right\|$,

$\left\|e_{r}\right\|=\left\|r^{m}\right\| /\left\|r^{o}\right\|$,

where $p^{m}, v^{m}$, and $r^{m}$ denote the pressure, velocity, and residual from m-MSFV; $p^{o}, v^{o}$, and $r^{o}$ denote pressure, velocity, and residual from original MSFV; $p^{f}$ and $v^{f}$ represent the fine-scale reference pressure and velocity. All the pressure plots are scaled by the boundary pressure condition. The local TPFA and LBC approaches are referred to as "m-MSFV(TPFA)" and "m-MSFV(LBC)", respectively.

\subsection{Case 1: SPE 10 bottom layer}

The first example is the SPE 10 bottom layer case with $220 \times 60$ fine cells and $22 \times 6$ coarse cells. The pressure is fixed at $(220,0)$ and $(0,60)$ with the non-dimensional values of 1 and 0 , respectively, and no-flow BC are specified on all the boundaries. The permeability and fine-scale reference pressure solution are shown in Fig 10. Since the problem is elliptic, the pressure should be bounded by the pressure values at boundaries (i.e., 0 and 1). However, as shown in Fig 11a, the original MSFV pressure exceeds these bounds at several locations, which indicates that the obtained solution is nonmonotone. A strictly monotone MSFV pressure can be obtained by using m-MSFV(TPFA), as shown in Fig 11b. In this case, the m-MSFV(LBC) can also reduce the level of nonmonotonicity significantly as shown in Fig. 11c; however, this approach does not guarantee that the solution is monotone. Figure 12 shows the streamlines associated with fine-scale pressure obtained using the original and monotone MSFV methods. As shown in Fig 12b, the nonphysical MSFV pressure leads to circulations in the velocity field, which can decrease the stability of the entire nonlinear simulation procedure. On the contrary, there exist no circulations in the velocity field reconstructed by the monotone MSFV pressure. In addition, as seen from the pressure errors, the m-MSFV method can deliver a monotone pressure solution without sacrificing accuracy. 


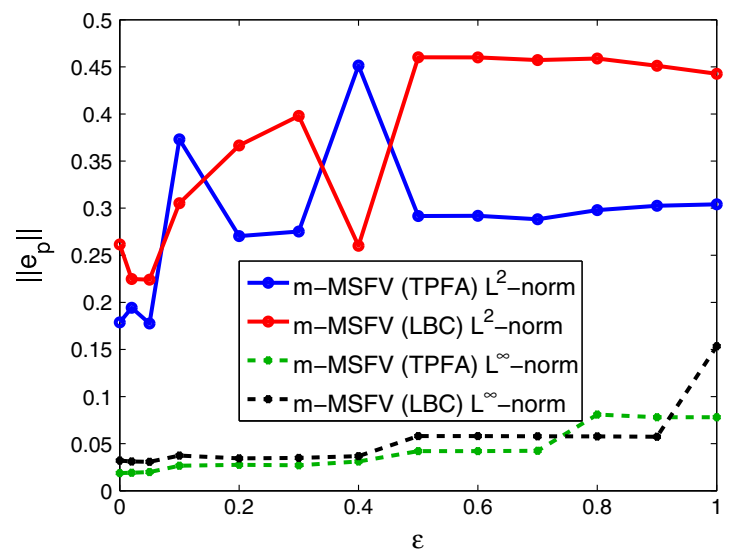

(a)

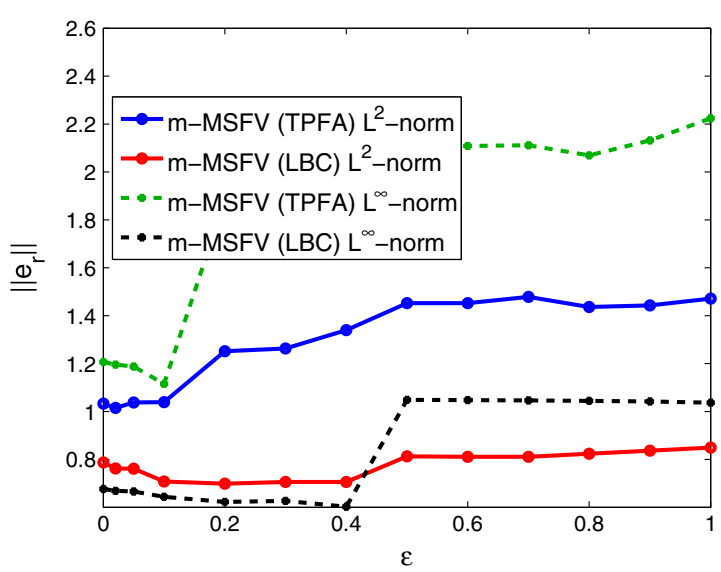

(c)

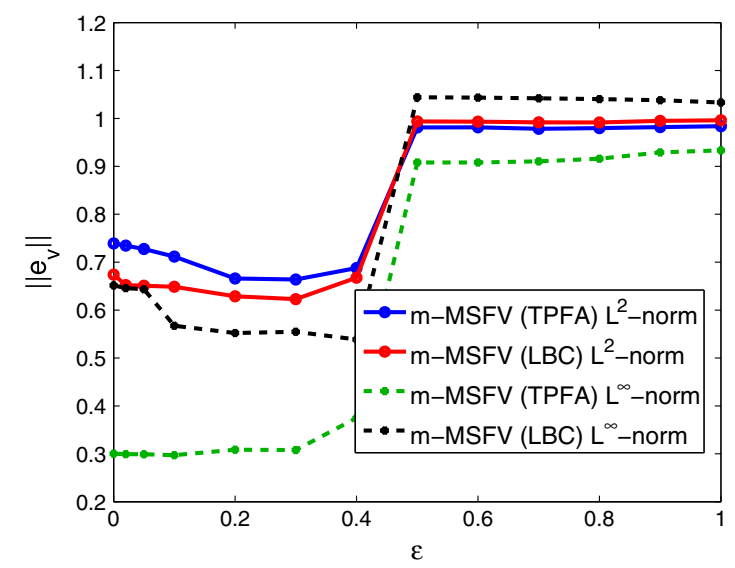

(b)

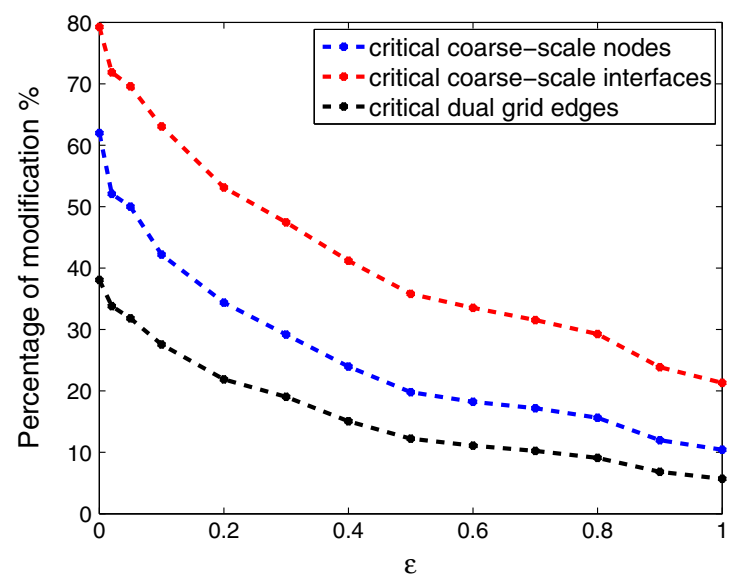

(d)

Fig. 15 Error measurements in pressure (a), velocity (b), residual (c), and the computational complexity (d) with different threshold $\varepsilon$ for the SPE 10 bottom layer

Figure 13 shows the histogram of $\eta_{i j}$ corresponding to the coarse-scale systems $A_{c}$ of the original MSFV, mMSFV(TPFA), and m-MSFV(LBC) methods. Note that the original coarse-scale system $A_{c}$ (Fig. 13a) has many positive indicators which span a wide range. These positive values lead to severely non-monotone pressure solution. With the modifications of m-MSFV(TPFA), the positive indicators are reduced to a limited range with small values, which are acceptable to obtain a monotone solution.
If zero indicators are desired, additional loops of detection and modification can be performed as described in Algorithm 1. On the other hand, with the modification of m-MSFV (LBC), even though this approach can eliminate some positive indicators, many areas with long-range indicator values still remain. These values may result in a non-monotone solution. Note that the remaining indicators cannot be eliminated by additional modification loops. That is the reason why m-MSFV(LBC) can reduce the level of

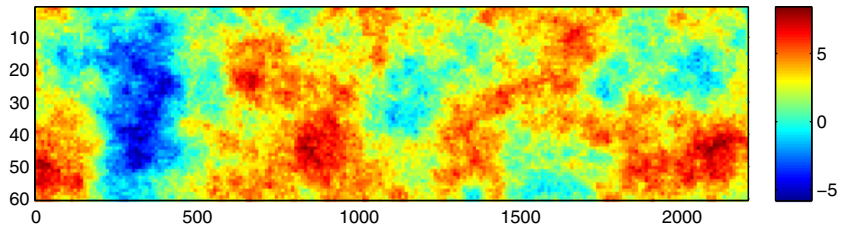

(a) Natural logarithm of the permeability

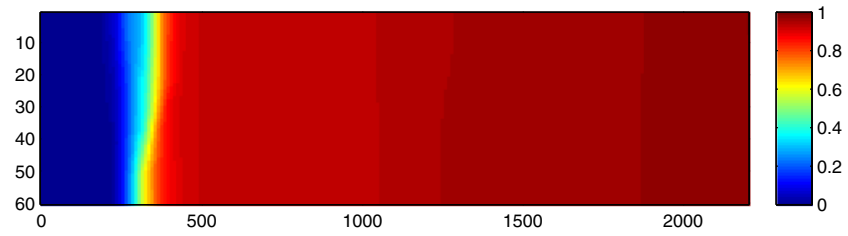

(b) Fine-scale reference pressure

Fig. 16 Permeability and fine-scale pressure solution for the SPE 10 top layer with stretched grids 


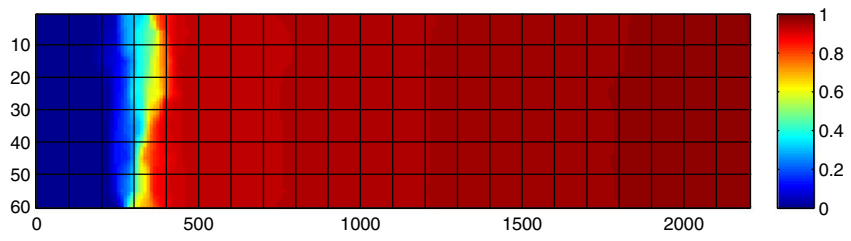

(a) Original $\operatorname{MSFV}\left(\left\|e_{p}\right\|_{2}=0.015 ;\left\|e_{p}\right\|_{\infty}=\right.$ 0.148 )

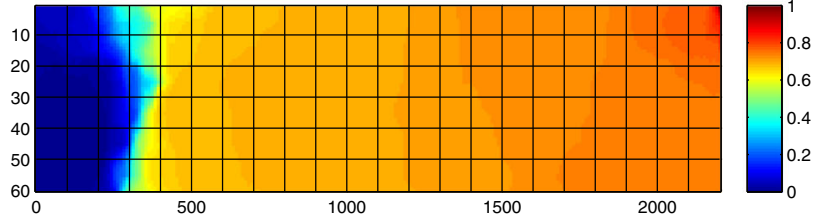

(b) $\mathrm{m}-\mathrm{MSFV}(\mathrm{TPFA})\left(\left\|e_{p}\right\|_{2}=0.252 ;\left\|e_{p}\right\|_{\infty}=\right.$ 0.407 )

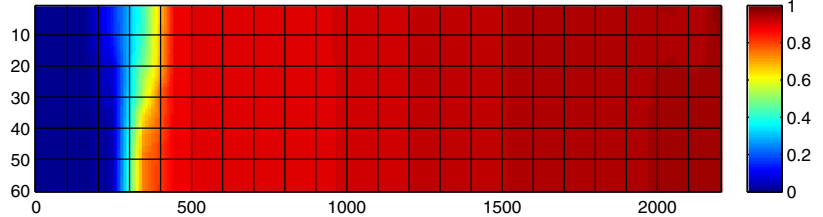

(c) $\mathrm{m}-\mathrm{MSFV}(\mathrm{LBC})\left(\left\|e_{p}\right\|_{2}=0.034 ;\left\|e_{p}\right\|_{\infty}=\right.$ $0.169)$

Fig. 17 Original MSFV and m-MSFV pressure solutions for the SPE 10 top layer with stretched grids, and the relative errors $e_{p}$

non-monotonicity, but cannot guarantee to fully resolve the issue for all the problems.

For practical purposes, strictly monotone pressure may not be required; therefore, the threshold value $\varepsilon$ provides a way to balance the degree of monotonicity and the computational cost of the m-MSFV method. Figure 14 shows that $\mathrm{m}$-MSFV (TPFA) with $\varepsilon=0$ guarantees that the pressure solution is strictly monotone. When the threshold is loosened to $\varepsilon=0.7$, the pressure solution still does not encounter severe non-monotone regions, while the computational effort is reduced by $50 \%$ compared with the $\varepsilon=0$ case. Figure 15 shows the accuracy of the $\mathrm{m}$-MSFV method with respect to different strategies and indicates that both m-MSFV(TPFA) and m-MSFV(LBC) have comparable error norms for pressure and velocity. The m-MSFV(LBC) approach results in slightly better residual estimates, since it preserves the MPFA stencil at coarse-scale, and just simplifies the heterogeneous field at the dual coarse cell boundaries.

\subsection{Case 2: SPE 10 layers with stretched grid}

In this case, both SPE 10 top and bottom layers with stretched grid are examined. The fine-scale and coarse-scale grids are $220 \times 60$ and $22 \times 6$, respectively. The global $\mathrm{BC}$ are the same as in Case 1. The fine-scale grid has an aspect ratio of 10, i.e., $\Delta x=10 \Delta y$. First, for SPE 10 top layer, the permeability field, fine-scale reference, original MSFV, and m-MSFV pressure solutions are shown in Figs. 16 and 17. Even though there are no significant peaks in the original MSFV pressure solution, the resulting streamlines of the original MSFV still have circulations. Also, in this case, the m-MSFV (TPFA) approach is using TPFA for almost the entire domain. Therefore, the pres-

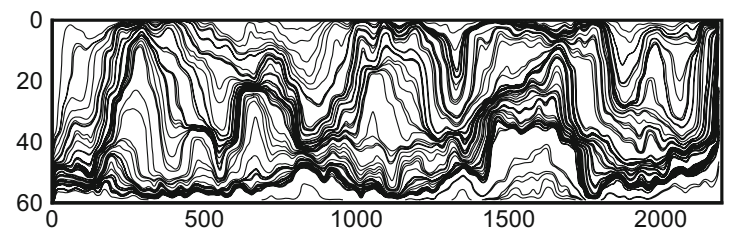

(a) Fine-scale reference

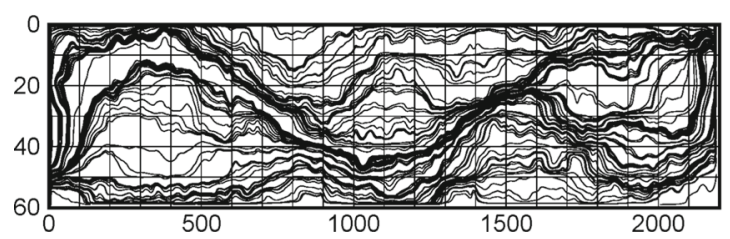

(c) m-MSFV (TPFA)

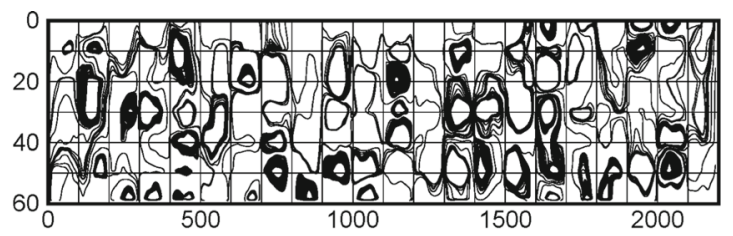

(b) Original MSFV

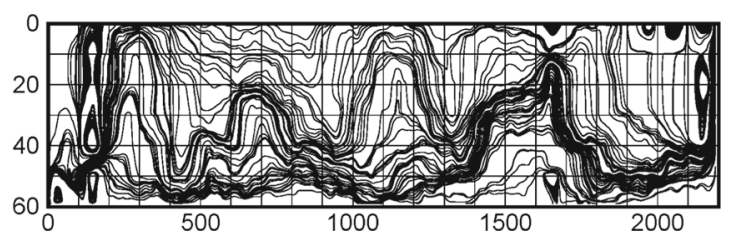

(d) m-MSFV (LBC)

Fig. 18 Streamline plots based on velocity fields reconstructed by fine-scale reference, original and monotone MSFV pressure solutions 
Fig. 19 Histogram of $\eta_{i j}$ of the coarse-scale system $A_{c}$ for original MSFV (a) and the reconstructed coarse-scale system for m-MSFV (LBC) (b), respectively, for the SPE 10 top layer with stretched grids. Note that m-MSFV (TPFA) eliminates all the positive indicators, therefore the histogram is not shown

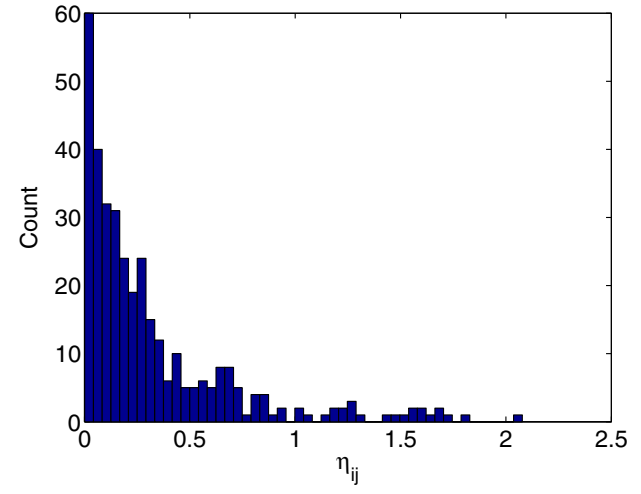

(a) Original coarse-scale system $A_{c}$

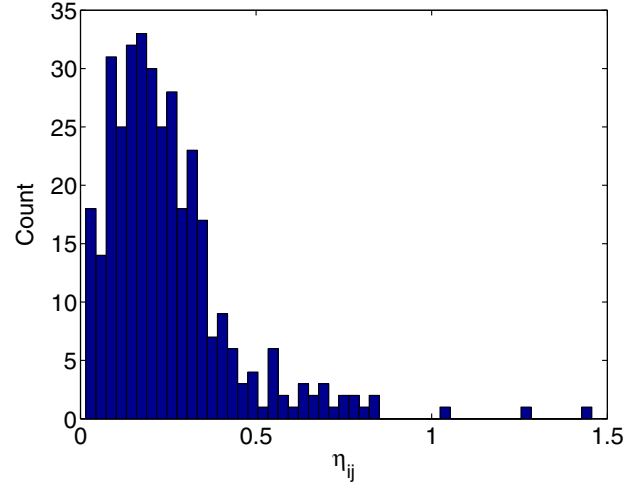

(b) Coarse-scale system $A_{c}$ with linear BC

sure solution is not accurate. However, m-MSFV (TPFA) can guarantee monotonicity of the pressure distribution, which can be indicated by the circulation-free streamlines (Fig. 18c). Circulations can be observed in the streamlines of m-MSFV (LBC) as shown in Fig. 18d, which implies that

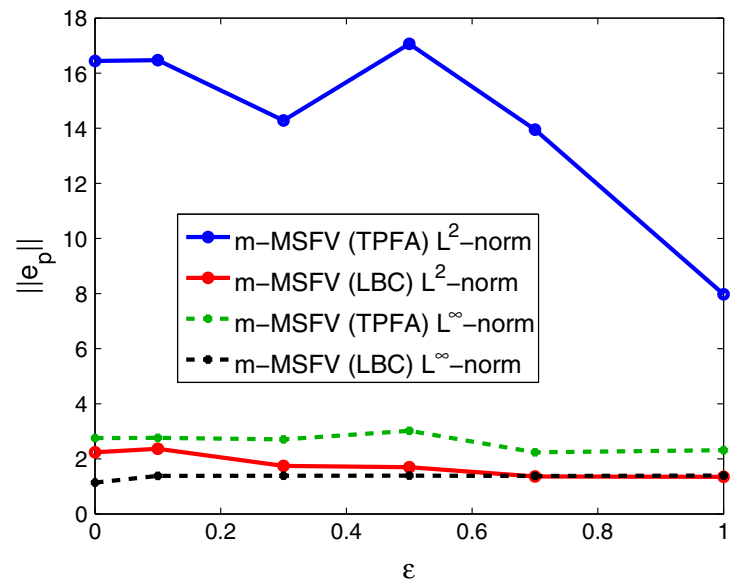

(a)

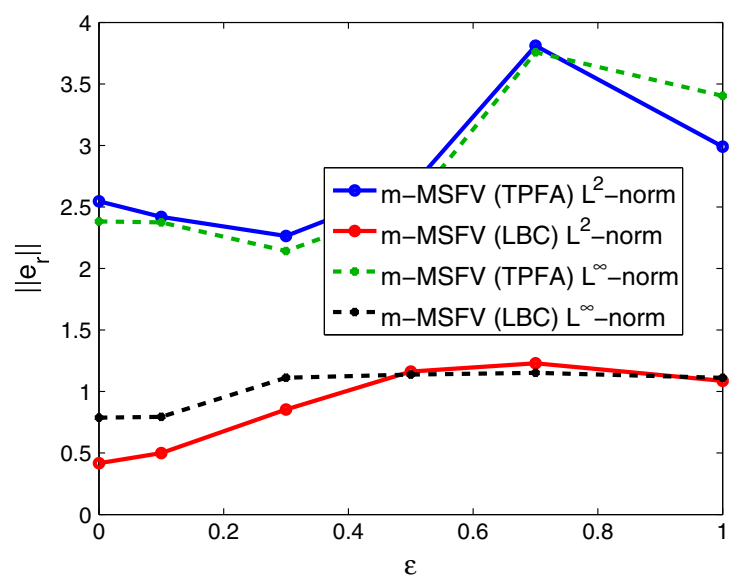

(c)
m-MSFV (LBC) cannot guarantee a monotone solution in this case. Moreover, the non-monotone solution for original MSFV and m-MSFV (LBC) can be identified by Fig. 19, which indicates that the long-range positive indicators of the coarse-scale system may lead to unphysical multiscale solutions.

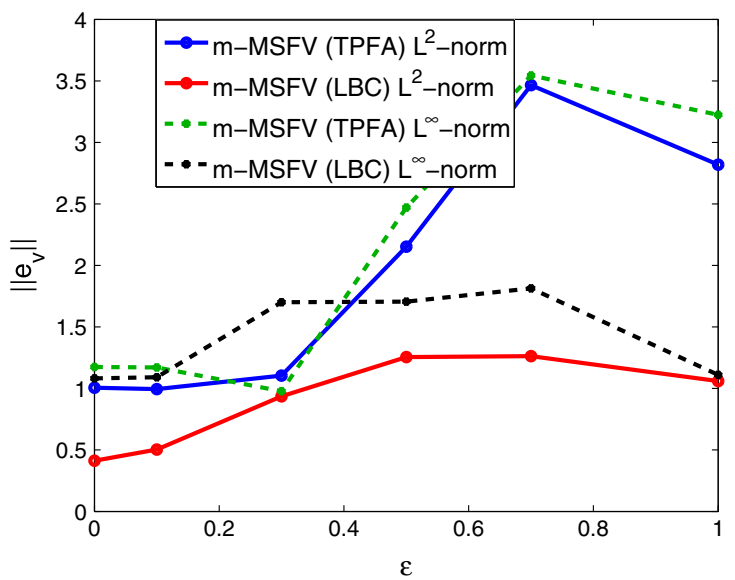

(b)

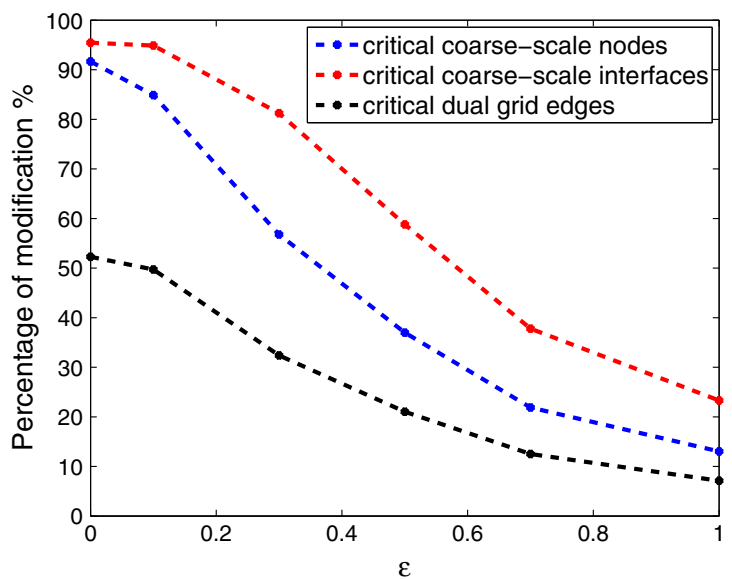

(d)

Fig. 20 Error measurements in pressure (a), velocity (b), residual (c) and computational complexity (d) with different threshold $\varepsilon$ for the SPE 10 top layer with stretched grids 


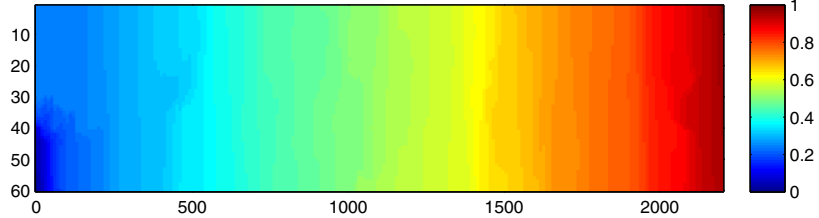

(a) Fine-scale reference

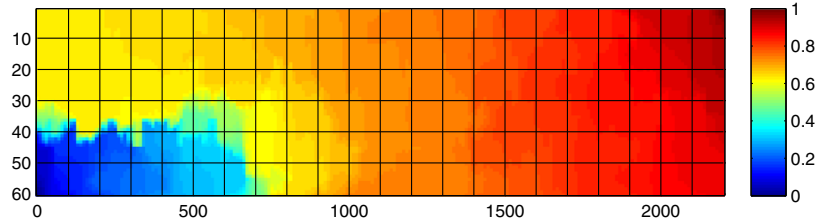

(c) $\mathrm{m}-\mathrm{MSFV}$ (TPFA) $\left(\left\|e_{p}\right\|_{2}=0.338 ;\left\|e_{p}\right\|_{\infty}=\right.$ $0.392)$

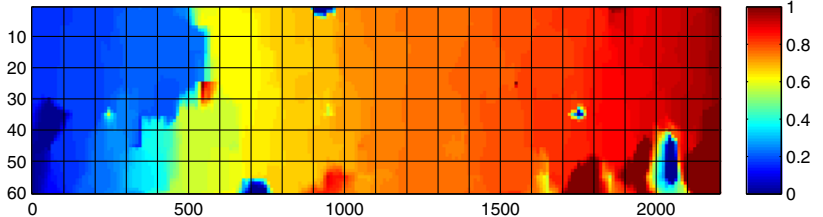

(b) Original $\operatorname{MSFV}\left(\left\|e_{p}\right\|_{2}=0.417 ;\left\|e_{p}\right\|_{\infty}=\right.$ 4.969)

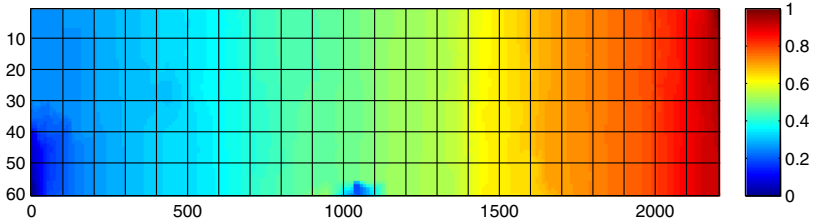

(d) $\mathrm{m}-\operatorname{MSFV}(\mathrm{LBC})\left(\left\|e_{p}\right\|_{2}=0.043 ;\left\|e_{p}\right\|_{\infty}=\right.$ $0.331)$

Fig. 21 Original MSFV and m-MSFV pressure solutions for the SPE 10 bottom layer with stretched grids, and the relative errors $e_{p}$

Similarly, as shown in Fig. 21, the original MSFV is severely nonmonotone for the SPE 10 bottom layer with stretched grids, and the m-MSFV (LBC) mitigates the issue. However, it cannot fully resolve it. The m-MSFV (TPFA) becomes a global TPFA scheme; therefore, it loses accuracy as indicated in the streamline plots shown in Fig. 22. In addition, Figs. 20 and 23 show the accuracy of the m-MSFV method with respect to different strategies and indicate that both m-MSFV(TPFA) and m-MSFV(LBC) have comparable error norms for pressure and velocity.

Note that the streamlines given by m-MSFV(LBC) honor the fine-scale reference quite well for the region where no circulations occur. Therefore, it is beneficial to apply m-MSFV(LBC) first, then employ m-MSFV(TPFA) for the places where m-MSFV(LBC) fails to resolve non- physical peaks. Hence, combining both m-MSFV(LBC) and m-MSFV(TPFA) can achieve circulation-free and conservative fine-scale velocity fields without losing accuracy for anisotropic problems. For the SPE 10 top layer with stretched grids, m-MSFV(LBC) is applied first resulting the pressure and velocity distributions as shown in Fig. 17c and 18d. From Fig. 18d, m-MSFV(LBC) cannot fully resolve the circulations for some particular regions but results in streamlines that are quite close to fine-scale reference in most regions. In order to remove the circulations, the m-MSFV(TPFA) approach can be employed for the regions where $\mathrm{m}-\mathrm{MSFV}$ (LBC) is not adequate. With the combination of both approaches, we can obtain the finescale pressure and velocity fields shown in Figs. 24 and 25. In additional, the pressure, velocity, and residual errors with

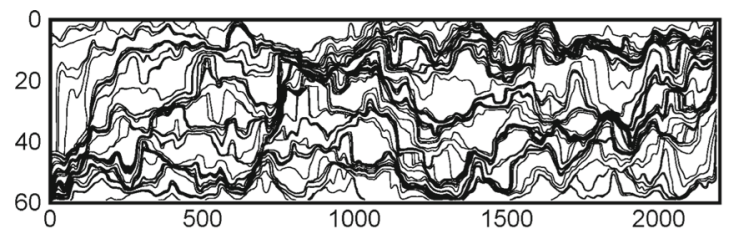

(a) Fine-scale reference

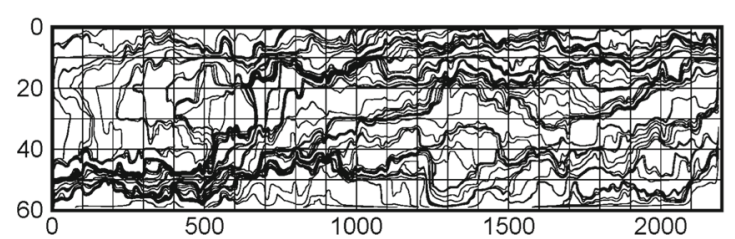

(c) m-MSFV (TPFA)

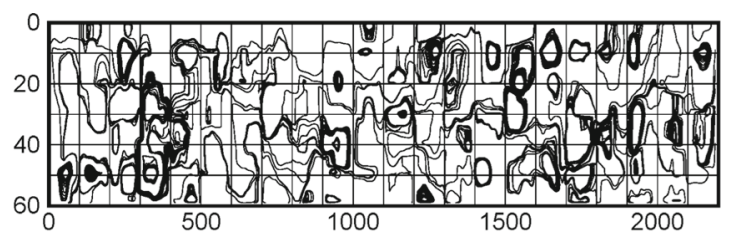

(b) Original MSFV

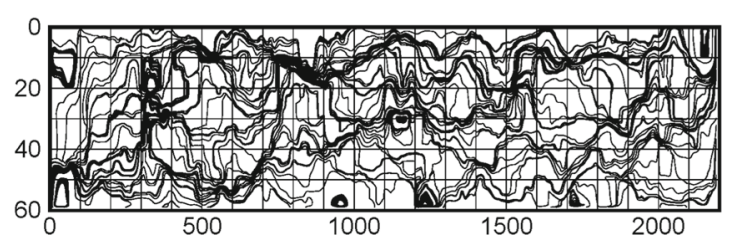

(d) m-MSFV (LBC)

Fig. 22 Streamline plots based on velocity fields reconstructed by fine-scale reference, original and m-MSFV pressure solutions for the SPE 10 bottom layer with stretched grids. The coarse-scale grids are indicated by black lines 


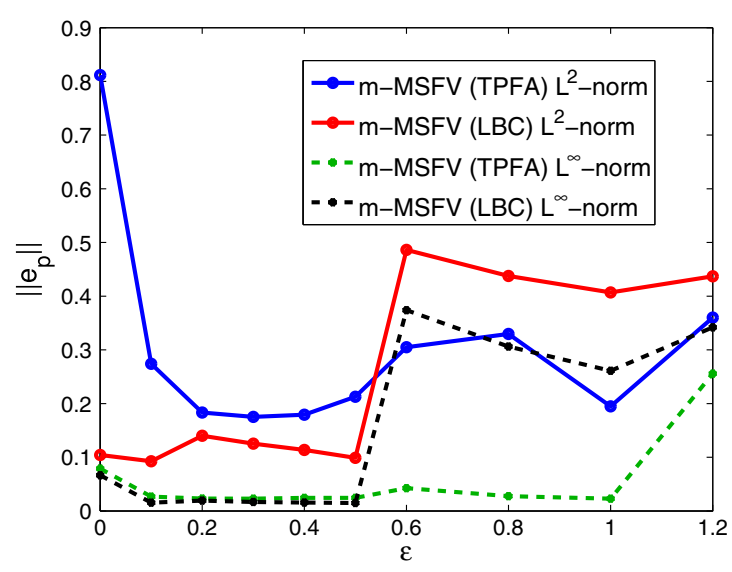

(a)

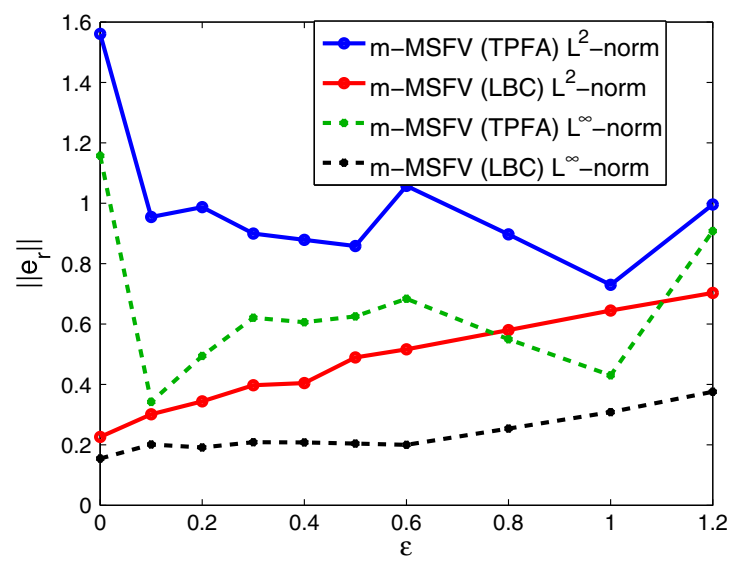

(c)

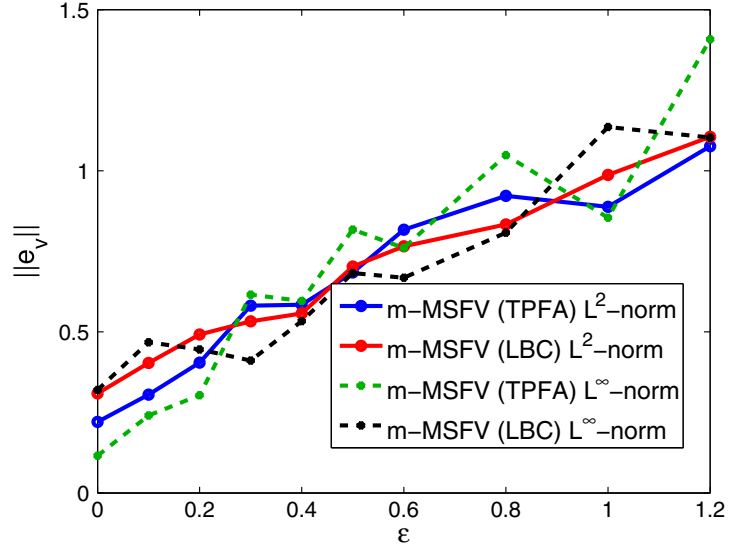

(b)

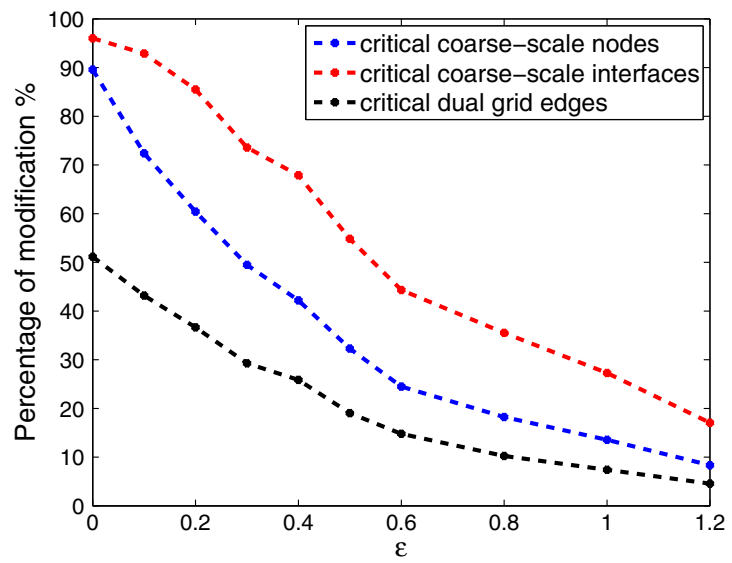

(d)

Fig. 23 Error measurements in pressure (a), velocity (b), residual (c), and computational complexity (d) with different threshold $\varepsilon$ for the SPE 10 bottom layer with stretched grids

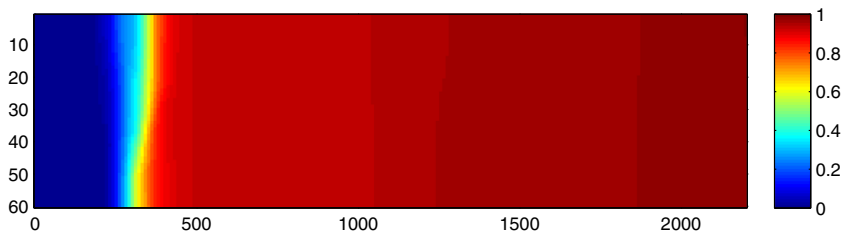

(a)

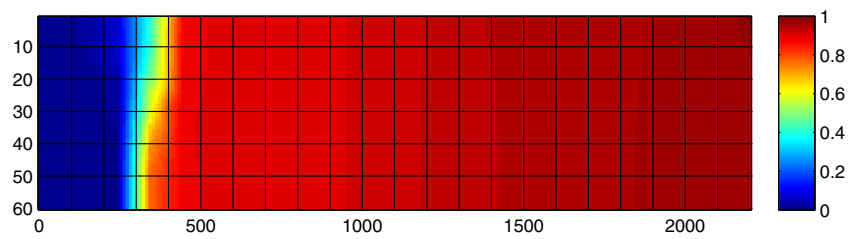

(b)

Fig. 24 Pressure distributions for fine-scale reference (a) and obtained by hybrid m-MSFV method (b) for the SPE 10 top layer with stretched grids, i.e., $\Delta x=10 \Delta y$

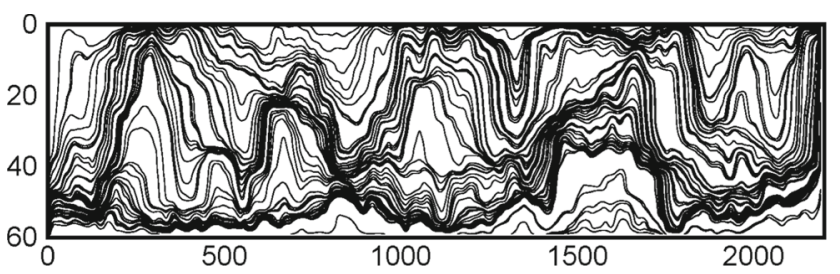

(a)

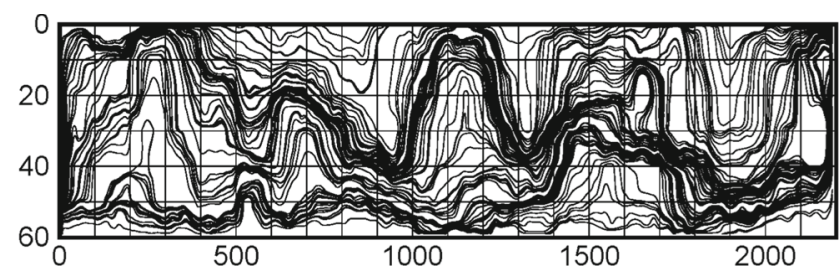

(b)

Fig. 25 Velocity distributions for fine-scale reference (a) and obtained by hybrid m-MSFV method (b) for the SPE 10 top layer with stretched grids, i.e., $\Delta x=10 \Delta y$ 
Table 1 Relative errors of hybrid m-MSFV, m-MSFV(TPFA), mMSFV(LBC), and original MSFV for the SPE 10 top layer with stretched grids, i.e., $\Delta x=10 \Delta y$. In addition, the last two columns represent the amount of TPFA coarse-scale interfaces and dual-grid boundaries using LBC for all the methods

\begin{tabular}{|c|c|c|c|c|c|c|c|c|}
\hline Error & $\left\|e_{p}\right\|_{2}$ & $\left\|e_{p}\right\|_{\infty}$ & $\left\|e_{v}\right\|_{2}$ & $\left\|e_{v}\right\|_{\infty}$ & $\left\|e_{r}\right\|_{2}$ & $\left\|e_{r}\right\|_{\infty}$ & interfaces & LBC \\
\hline Hybrid m-MSFV & 0.034 & 0.187 & 0.926 & 0.585 & 0.059 & 0.019 & $14 \%$ & $33 \%$ \\
\hline m-MSFV(TPFA) & 0.252 & 0.407 & 8.165 & 8.816 & 0.309 & 0.052 & $95 \%$ & - \\
\hline m-MSFV(LBC) & 0.034 & 0.169 & 3.349 & 8.119 & 0.051 & 0.017 & - & $52 \%$ \\
\hline original MSFV & 0.015 & 0.148 & 8.115 & 7.507 & 0.122 & 0.022 & - & - \\
\hline
\end{tabular}

respect to the fine-scale reference are given in Table 1, where we can see that the hybrid m-MSFV delivers the most accurate velocity field.

\section{Conclusions}

In this paper, a monotone MultiScale Finite Volume (mMSFV) method was proposed. The m-MSFV is based on automatic detection of the local interfaces with negative coarse-scale transmissibilities obtained from the integration of fluxes induced by the dual basis functions. Two approaches were developed to fix the non-physical coarsescale transmissibility, namely, local TPFA and local linear BC approaches. For the first approach, a local TPFA method for the critical interfaces only is used to calculate a positive transmissibility and replace the original MPFA stencils on the coarse-scale system. For the second approach, a linear $\mathrm{BC}$ is employed as the local boundary assumption to solve the basis function only for the dual-coarse cells associated with the critical coarse nodes. Then, the coarse-scale system is reconstructed and solved. The local TPFA approach can guarantee monotonicity of the reconstructed fine-scale solution. The local linear BC can mitigate the level of nonmonotonicity, but without a guarantee to remove all local pressure oscillations. Therefore, a hybrid strategy that combines both approaches may be effective, whereby the local linear BC approach is used to reduce the degree of nonmonotonicity and local TPFA approach is used to achieve the monotonicity for the regions where the linear BC cannot help. Since this m-MSFV method only employs a local fix for critical coarse-cell interfaces that lie in low-permeability regions, the transmissibility values have a small impact on the flow activity. This helps the m-MSFV solution be quite accurate with respect to the fine-scale reference. Moreover, the m-MSFV method is able to optimize the efficiencymonotonicity tradeoff. Finally, using the m-MSFV method is expected to improve the overall efficiency of sequential fully implicit simulations, which is the focus of our current research.
Acknowledgments The authors acknowledge financial support from the Petroleum Institute (PI) and the Abu Dhabi National Oil Company (ADNOC).

Open Access This article is distributed under the terms of the Creative Commons Attribution 4.0 International License (http:// creativecommons.org/licenses/by/4.0/), which permits unrestricted use, distribution, and reproduction in any medium, provided you give appropriate credit to the original author(s) and the source, provide a link to the Creative Commons license, and indicate if changes were made.

\section{References}

1. Hou, T., Wu, X.H.: A multiscale finite element method for elliptic problems in composite matrials and porous meida. J. Comput. Phys. 134, 169-189 (1997)

2. Efendiev, Y., Ginting, V., Hou, T., Ewing, R.: Convergence of a nonconforming multiscale finite element method. SIAM J. Numer. Anal. 37(3), 888-910 (2000)

3. Aarnes, J., Hou, T.Y.: Multiscale domain decomposition methods for elliptic problems with high aspect ratios. Acta Math. Appl. 18(1), 63-76 (2002)

4. Arbogast, T., Bryant, S.L.: A two-scale numerical subgrid technique for waterflood simulations. SPE J. 7, 446-457 (2002)

5. Chen, Z., Hou, T.: A mixed finite element method for elliptic problems with rapidly oscillating coefficients. Math. Comput. 72, 541$576(2003)$

6. Jenny, P., Lee, S.H., Tchelepi, H.A.: Multi-scale finite-volume method for elliptic problems in subsurface flow simulation. J. Comput. Phys. 187, 47-67 (2003)

7. Aarnes, J.E.: On the use of a mixed multiscale finite element method for greater flexibility and increased speed or improved accuracy in reservoir simulation. Multiscale Model. Simul. 2(3), 421-439 (2004)

8. Aarnes, J.E., Kippe, V., Lie, K.-A.: Mixed multiscale finite elements and streamline methods for reservoir simulation of large geomodels. Adv. Water Resour. 28(3), 257-271 (2005)

9. Efendiev, Y., Hou, T.Y.: Multiscale Finite Element Methods: Theory and Applications. Springer (2009)

10. Jenny, P., Lee, S.H., Tchelepi, H.A.: Adaptive fully implicit multiscale finite-volume method for multi-phase flow and transport in heterogeneous porous media. J. Comput. Phys. 217, 627-641 (2006) 
11. Lee, S.H., Wolfsteiner, C., Tchelepi, H.A.: Multiscale finitevolume formulation for multiphase flow in porous media: Black oil formulation of compressible, three-phase flow with gravity. Comput. Geosci. 12(3), 351-366 (2008)

12. Zhou, H., Lee, S.H., Tchelepi, H.A.: Multiscale finite-volume formulation for saturation equations. SPE J. 17(1), 198-211 (2011)

13. Zhou, H., Tchelepi, H.A.: Operator-based multiscale method for compressible flow. SPE J. 13, 267-273 (2008)

14. Hajibeygi, H., Jenny, P.: Multiscale finite-volume method for parabolic problems arising from compressible multiphase flow in porous media. J. Comput. Phys. 228, 5129-5147 (2009)

15. Hajibeygi, H., Tchelepi, H.A.: Compositional multiscale finitevolume formulation. SPE J. 19(2), 316-326 (2014)

16. Hajibeygi, H., Karvounis, D., Jenny, P.: A hierarchical fracture model for the iterative multiscale finite volume method. J. Comput. Phys. 230(24), 8729-8743 (2011)

17. Wolfsteiner, C., Lee, S.H., Tchelepi, H.A.: Well modeling in the multiscale finite volume method for subsurface flow simulation. SIAM Multiscale Model. Simul. 5(3), 900-917 (2006)

18. Jenny, P., Lunati, I.: Modeling complex wells with the multiscale finite volume method. J. Comput. Phys. 228, 687-702 (2009)

19. Lee, S.H., Zhou, H., Tchelepi, H.A.: Adaptive multiscale finitevolume method for nonlinear multiphase transport in heterogeneous formations. J. Comput. Phys. 228(24), 9036-9058 (2009)
20. Hajibeygi, H., Lee, S.H., Lunati, I.: Accurate and efficient simulation of multiphase flow in a heterogeneous reservoir by using error estimate and control in the multiscale finite-volume framework. SPE J. 17(4), 1071-1083 (2012)

21. Hajibeygi, H., Bonfigli, G., Hesse, M.A., Jenny, P.: Iterative multiscale finite volume method. J. Comput. Phys. 227, 8604-8621 (2008)

22. Zhou, H., Tchelepi, H.A.: Two-stage algebraic multiscale linear solver for highly heterogeneous reservoir models. SPE J. 17(2), 523-539 (2012)

23. Wang, Y., Hajibeygi, H., Tchelepi, H.A.: Algebraic multiscale solver for flow in heterogeneous porous media. J. Comput. Phys. 259, 284-303 (2014)

24. Hajibeygi, H., Jenny, P.: Adaptive iterative multiscale finite volume method. J. Comput. Phys. 230, 628-643 (2011)

25. Hesse, M.A., Mallison, B.T., Tchelepi, H.A.: Compact multiscale finite volume method for heterogeneous anisotropic elliptic equations. SIAM Multiscale Model. Simul. 7, 934-962 (2008)

26. Lunati, I., Jenny, P.: Treating highly anisotropic subsurface flow with the multiscale finite-volume method. SIAM Multiscale Model. Simul. 6(1), 308-318 (2007)

27. Aziz, K., Settari, A.: Petroleum reservoir simulation. Blitzprint Ltd. Cagary, Alberta (2002)

28. Christie, M.A., Blunt, M.J.: Tenth SPE comparative solution project: a comparison of upscaling techniques. SPE Reserv. Eval. Eng. 4, 308-317 (2001) 